\title{
Polyprenylated Benzoylphloroglucinols with DNA Polymerases Inhibitory Activity from the Fruits of Garcinia schomburgkiana
}

Duy Hoang Le, ${ }^{\dagger}$ Katsumi Nishimura, ${ }^{\dagger}$ Yukiko Takenaka,${ }^{\dagger}$ Yoshiyuki Mizushina, ${ }^{\dagger}$ and Takao Tanahashi ${ }^{*}, \dagger$

${ }^{\dagger}$ Department of Organic chemistry, Kobe Pharmaceutical University, Kobe 658-8558, Japan

$\ddagger$ Graduate School of Agriculture, Shinshu University, Kamiina-gun, Nagano 399-4598, Japan 


\section{List of content}

Figure S1. ECD spectra of 1-3, 9, and $\mathbf{1 0}$

Figure S2. ECD spectra of 4-7

Figure S3. Structure of 1a'; Spectroscopic data of 1a'

Figure S4. IR and MS spectra of 1

Figure S5. ${ }^{1} \mathrm{H}$ NMR spectrum (500 MHz, methanol- $d_{4}+0.1 \%$ TFA- $d$ ) of 1

Figure S6. ${ }^{13} \mathrm{C}$ NMR spectrum $\left(125 \mathrm{MHz}\right.$, methanol- $d_{4}+0.1 \%$ TFA- $d$ ) of 1

Figure S7. IR and MS spectra of 2

Figure S8. ${ }^{1} \mathrm{H}$ NMR spectrum (500 MHz, methanol- $d_{4}+0.1 \%$ TFA- $d$ ) of 2

Figure S9. ${ }^{13} \mathrm{C}$ NMR spectrum $\left(125 \mathrm{MHz}\right.$, methanol- $d_{4}+0.1 \%$ TFA- $d$ ) of 2

Figure S10. IR and MS spectra of 3

Figure S11. ${ }^{1} \mathrm{H}$ NMR spectrum (500 MHz, methanol- $d_{4}+0.1 \%$ TFA- $d$ ) of 3

Figure S12. ${ }^{13} \mathrm{C}$ NMR spectrum ( $125 \mathrm{MHz}$, methanol- $d_{4}+0.1 \%$ TFA- $d$ ) of 3

Figure S13. IR and MS spectra of 4

Figure S14. ${ }^{1} \mathrm{H}$ NMR spectrum $\left(500 \mathrm{MHz}, \mathrm{CDCl}_{3}\right)$ of 4

Figure S15. ${ }^{13} \mathrm{C}$ NMR spectrum $\left(125 \mathrm{MHz}, \mathrm{CDCl}_{3}\right)$ of 4

Figure S16. IR and MS spectra of 5

Figure S17. ${ }^{1} \mathrm{H}$ NMR spectrum $\left(500 \mathrm{MHz}, \mathrm{CDCl}_{3}\right)$ of $\mathbf{5}$

Figure S18. ${ }^{13} \mathrm{C}$ NMR spectrum $\left(125 \mathrm{MHz}, \mathrm{CDCl}_{3}\right)$ of $\mathbf{5}$

Figure S19. IR and MS spectra of 6

Figure S20. ${ }^{1} \mathrm{H}$ NMR spectrum $\left(500 \mathrm{MHz}\right.$, methanol- $d_{4}+0.1 \%$ TFA- $d$ ) of 6

Figure S21. ${ }^{13} \mathrm{C}$ NMR spectrum (125 MHz, methanol- $d_{4}+0.1 \%$ TFA- $d$ ) of 6

Figure S22. IR and MS spectra of 7

Figure S23. ${ }^{1} \mathrm{H}$ NMR spectrum (500 MHz, methanol- $d_{4}+0.1 \%$ TFA- $d$ ) of 7

Figure S24. ${ }^{13} \mathrm{C}$ NMR spectrum (125 MHz, methanol- $d_{4}+0.1 \%$ TFA- $d$ ) of 7

Figure S25. IR and MS spectra of 8

Figure S26. ${ }^{1} \mathrm{H}$ NMR spectrum (500 MHz, methanol- $d_{4}+0.1 \%$ TFA- $d$ ) of 8

Figure S27. ${ }^{13} \mathrm{C}$ NMR spectrum (125 MHz, methanol- $d_{4}+0.1 \%$ TFA- $d$ ) of 8 


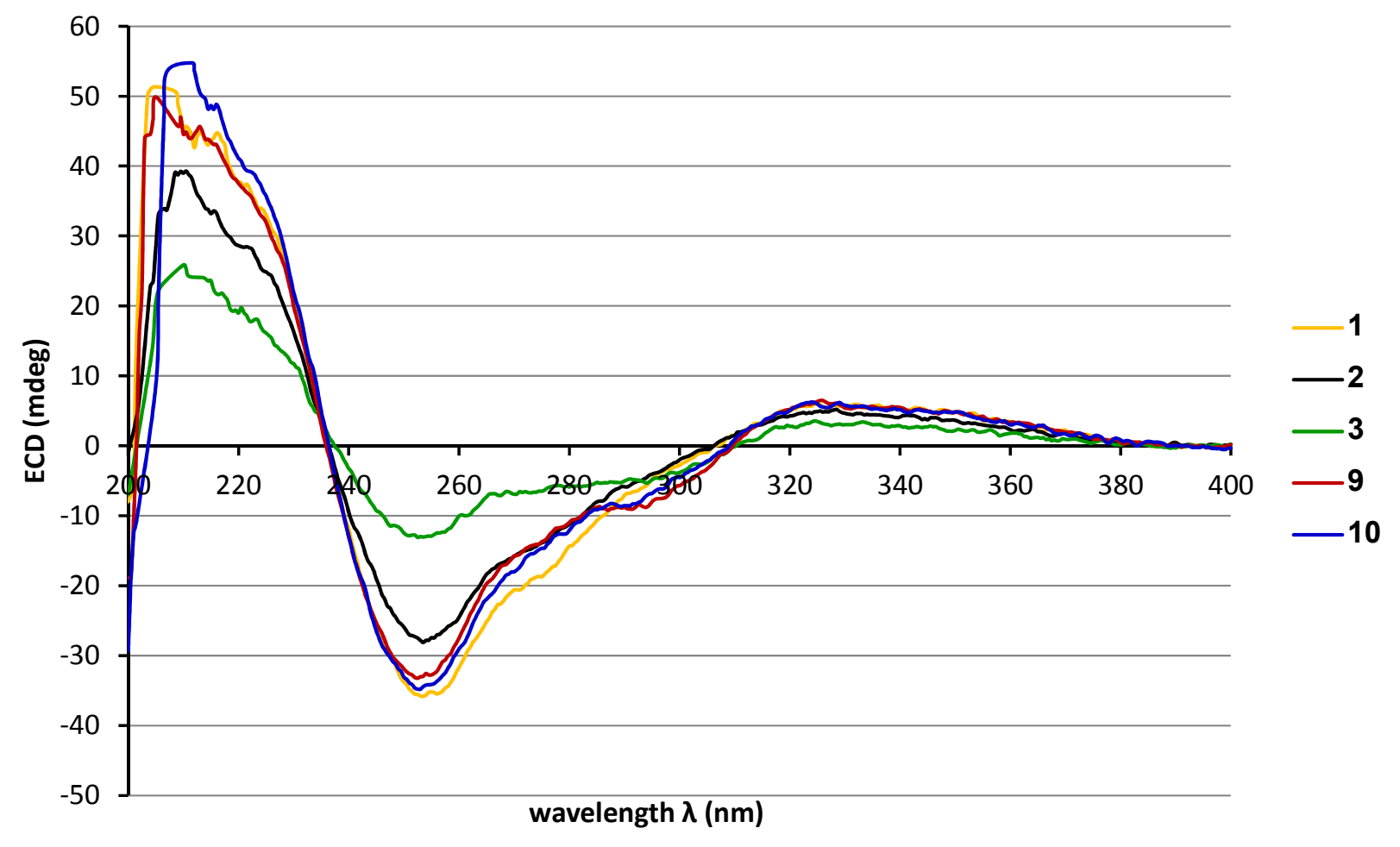

Figure S1. ECD spectra of 1-3, 9, and $\mathbf{1 0}$

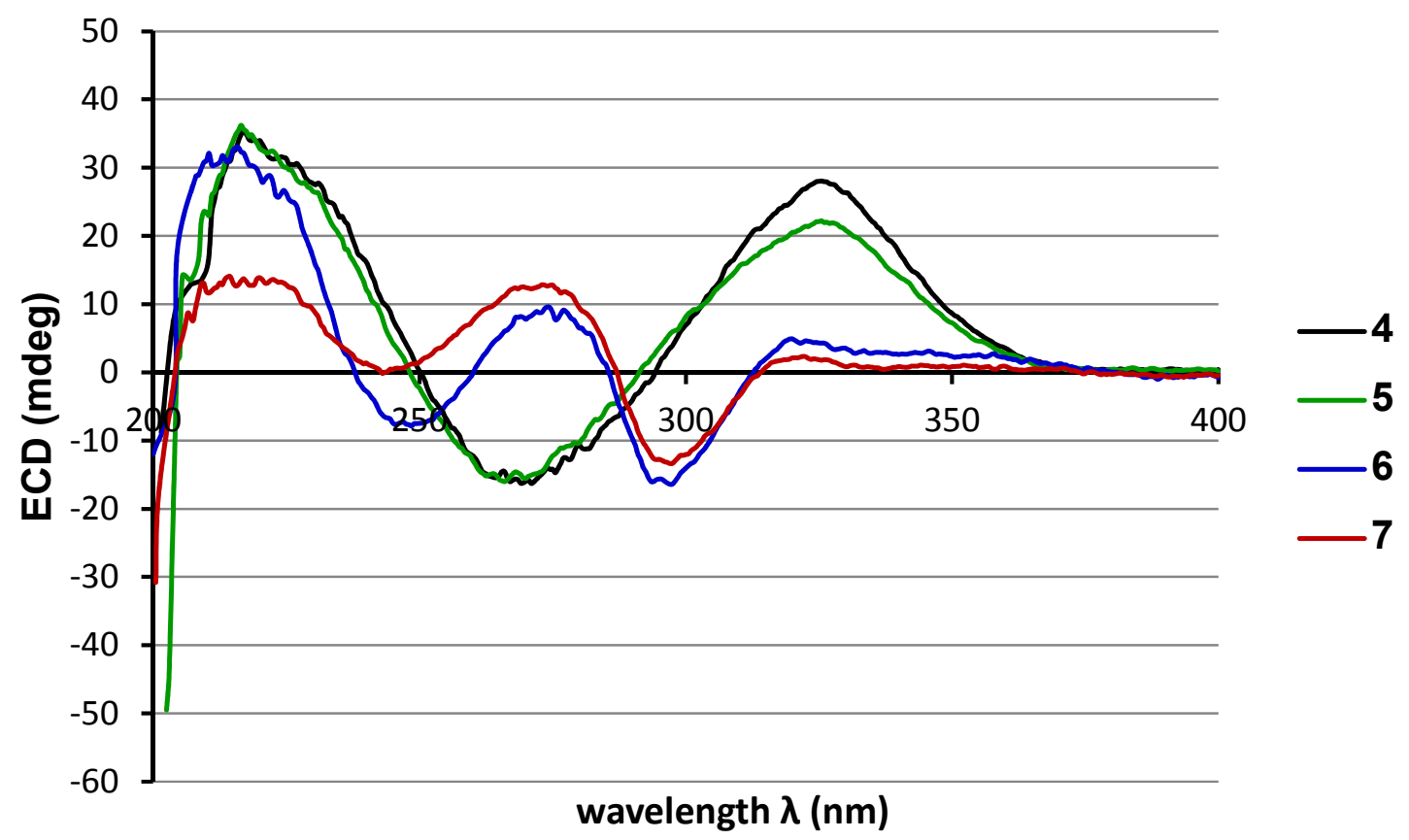

Figure S2. ECD spectra of 4-7 


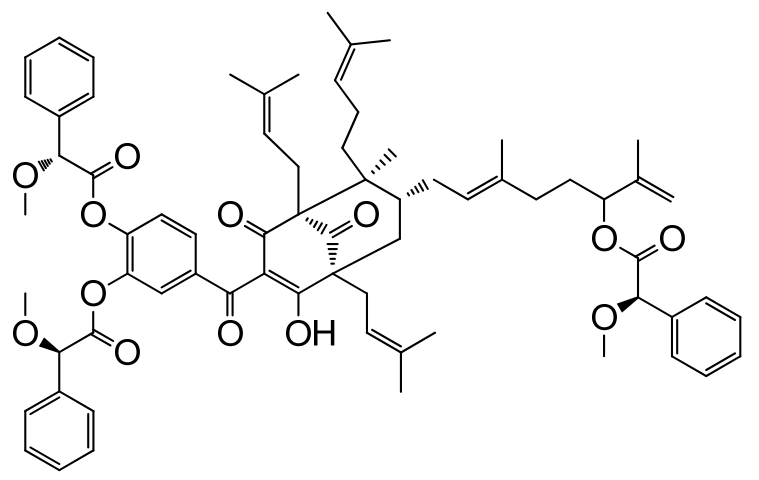

Figure S3. Structure of $1 \mathbf{a}^{\prime}$

\section{Spectroscopic data of 1a':}

${ }^{1} \mathrm{H}$ NMR $\left(500 \mathrm{MHz}\right.$, methanol- $d_{4}+0.1 \%$ TFA-d) $\delta_{\mathrm{H}} 7.528-7.515(17 \mathrm{H}, \mathrm{m}, \mathrm{H}-12, \mathrm{H}-16,3 \times \mathrm{Ph})$, $7.016(1 \mathrm{H}, \mathrm{d}, J=8.5 \mathrm{~Hz}, \mathrm{H}-15), 5.081(1 \mathrm{H}$, brt, $J=5.5 \mathrm{~Hz}, \mathrm{H}-30), 5.050$ (2H, t, $J=7.0 \mathrm{~Hz}, \mathrm{H}-35, \mathrm{H}-$ 40), 4.829 (1H, br, H-18), 4.790 (1H, m, H-25), 4.804, 4.782, 4.622 (each 1H, s, 3×MPA-C $\underline{\mathrm{H}}<$ ), 4.664, 4.622 (each 1H, br s, $\mathrm{H}_{2}-42$ ), 3.395, 3.383, 3.297 (each 3H, s, 3×MPA-OC$\left.\underline{H}_{3}\right), 2.710(1 \mathrm{H}$, dd, $J=15.0,7.5 \mathrm{~Hz}, \mathrm{H}-17), 2.630$ (1H, br d, $J=15.0 \mathrm{~Hz}, \mathrm{H}-17), 2.510$ (1H, dd, $J=14.0,9.0 \mathrm{~Hz}, \mathrm{H}-29$ ), 2.440 (1H, br d, $J=14.0$ Hz, H-29), 2.040, 1.740 (each 1H, m, $\left.\mathrm{H}_{2}-24\right), 1.995,1.450$ (each 1H, m, $\mathrm{H}_{2-}$ 7), 1.940 (2H, m, H $\left.{ }_{2}-34\right), 1.875$ (2H, m, H $\left.{ }_{2}-27\right), 1.720$ (1H, m, H-6), 1.660 (2H, m, H (2H, m, H $\left.{ }_{2}-23\right), 1.675\left(3 \mathrm{H}, \mathrm{s}, \mathrm{H}_{3}-33\right), 1.672\left(3 \mathrm{H}, \mathrm{s}, \mathrm{H}_{3}-38\right), 1.655\left(3 \mathrm{H}, \mathrm{s}, \mathrm{H}_{3}-32\right), 1.641\left(3 \mathrm{H}, \mathrm{s}, \mathrm{H}_{3}-\right.$ 20), $1.584\left(3 \mathrm{H}, \mathrm{s}, \mathrm{H}_{3}-37\right), 1.564\left(3 \mathrm{H}, \mathrm{s}, \mathrm{H}_{3}-21\right), 1.519$ (3H, s, $\left.\mathrm{H}_{3}-28\right), 1.436\left(3 \mathrm{H}, \mathrm{s}, \mathrm{H}_{3}-43\right), 0.806$ $\left(3 \mathrm{H}, \mathrm{s}, \mathrm{H}_{3}-22\right)$.

${ }^{13} \mathrm{C}$ NMR (125 MHz, methanol- $d_{4}+0.1 \%$ TFA- $d$ ) $\delta_{\mathrm{C}} 196.3(\mathrm{C}-10), 171.6,169.4,169.2$ (3×MPA-COO), 144.2 (C-41), 137.9, 137.8, 137.1 (Ph), 137.0 (C-26), $136.8(\mathrm{Ph}), 135.5$ (C-31), 135.1 (C-19), 130.3, 130.0, 129.97, 129.8, 129.6, 128.6, 128.59, 128.5, 128.3, 128.1 (Ph), 125.3 (C35), $124.8(\mathrm{Ph}), 124.5$ (C-25), 123.5, 121.7 (Ph), 121.1 (C-18), 120.9 (C-30), 119.9, 119.2, 117.7, 117.2, 115.4, $114.9(\mathrm{Ph}), 113.4(\mathrm{C}-42), 111.4(\mathrm{Ph}), 83.9,82.9,82.8(3 \times \mathrm{MPA}-\underline{\mathrm{C}}<)$, $78.3(\mathrm{C}-40)$, 69.3 (C-4), 57.81, 57.80, 57.6 (3×MPA-O $\left.\underline{\mathrm{MH}}_{3}\right), 51.7$ (C-5), 43.0 (C-7), 41.7 (C-6), 37.4 (C-23), 36.2 (C-27), 31.8 (C-29, C-39), 29.9 (C-24), 26.5 (C-33), 26.4 (C-17), 26.3 (C-21), 25.9 (C-38), 25.2 (C-34), 18.3 (C-20), 18.1 (C-43), 18.0 (C-37), 16.2 (C-22), 16.2 (C-28).

HRESIMS $m / z 1153.5636[\mathrm{M}+\mathrm{Na}]^{+}$(calcd for $\mathrm{C}_{70} \mathrm{H}_{82} \mathrm{O}_{13} \mathrm{Na}, 1153.5657$ ). 

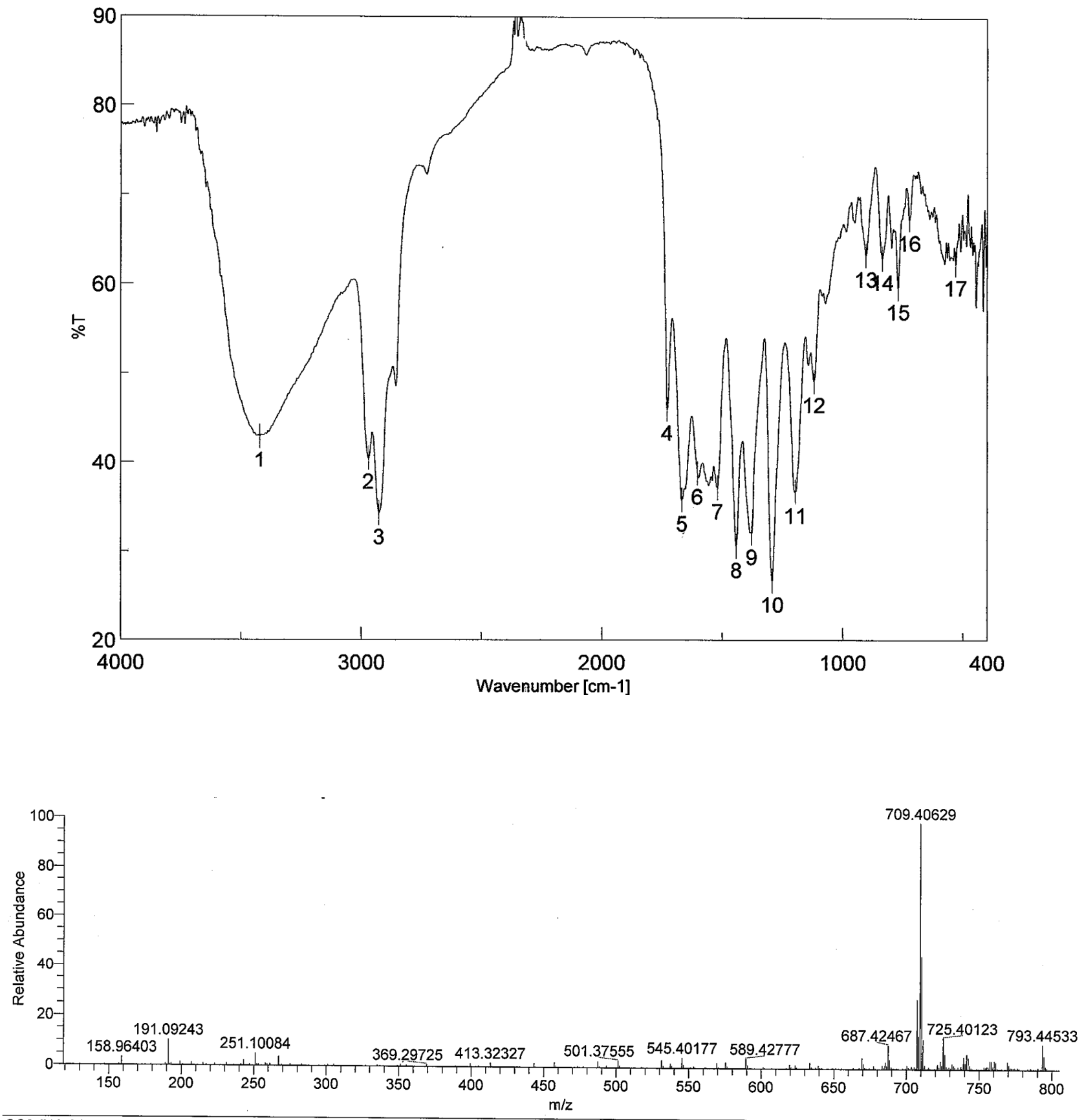

GS9 \#16-21 RT: 0.38-0.46 AV: 3 SB: 8 0.69-1.04 NL: 4.54E4 T: FTMS $\{1,1\}+p$ ESI Full ms [120.00-1000.00]

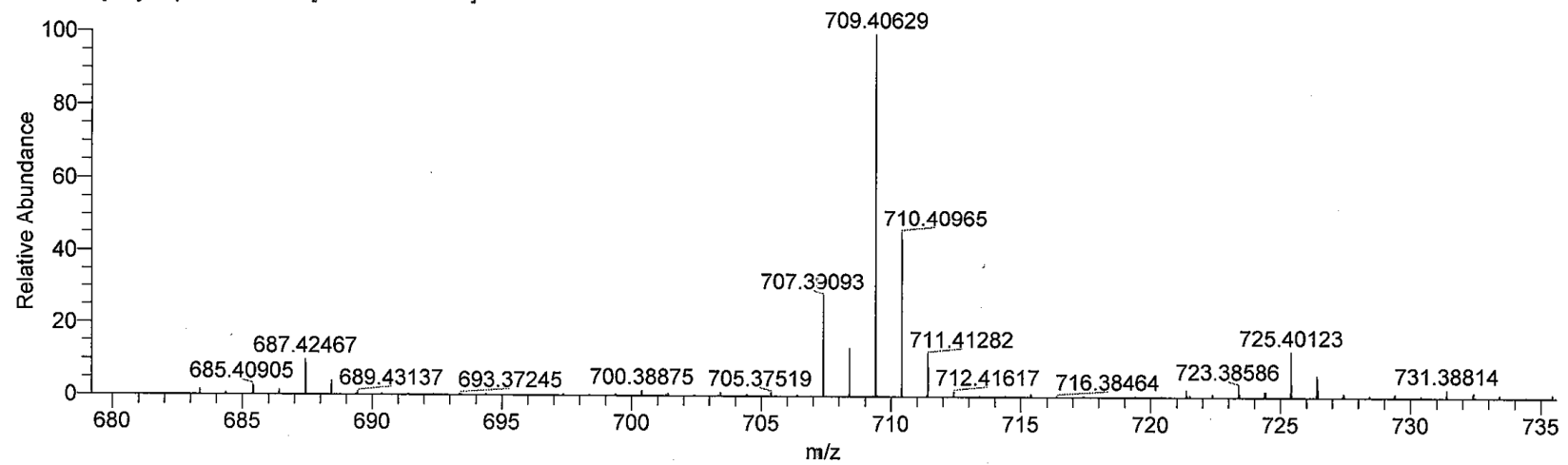

Figure S4. IR and MS spectra of 1 


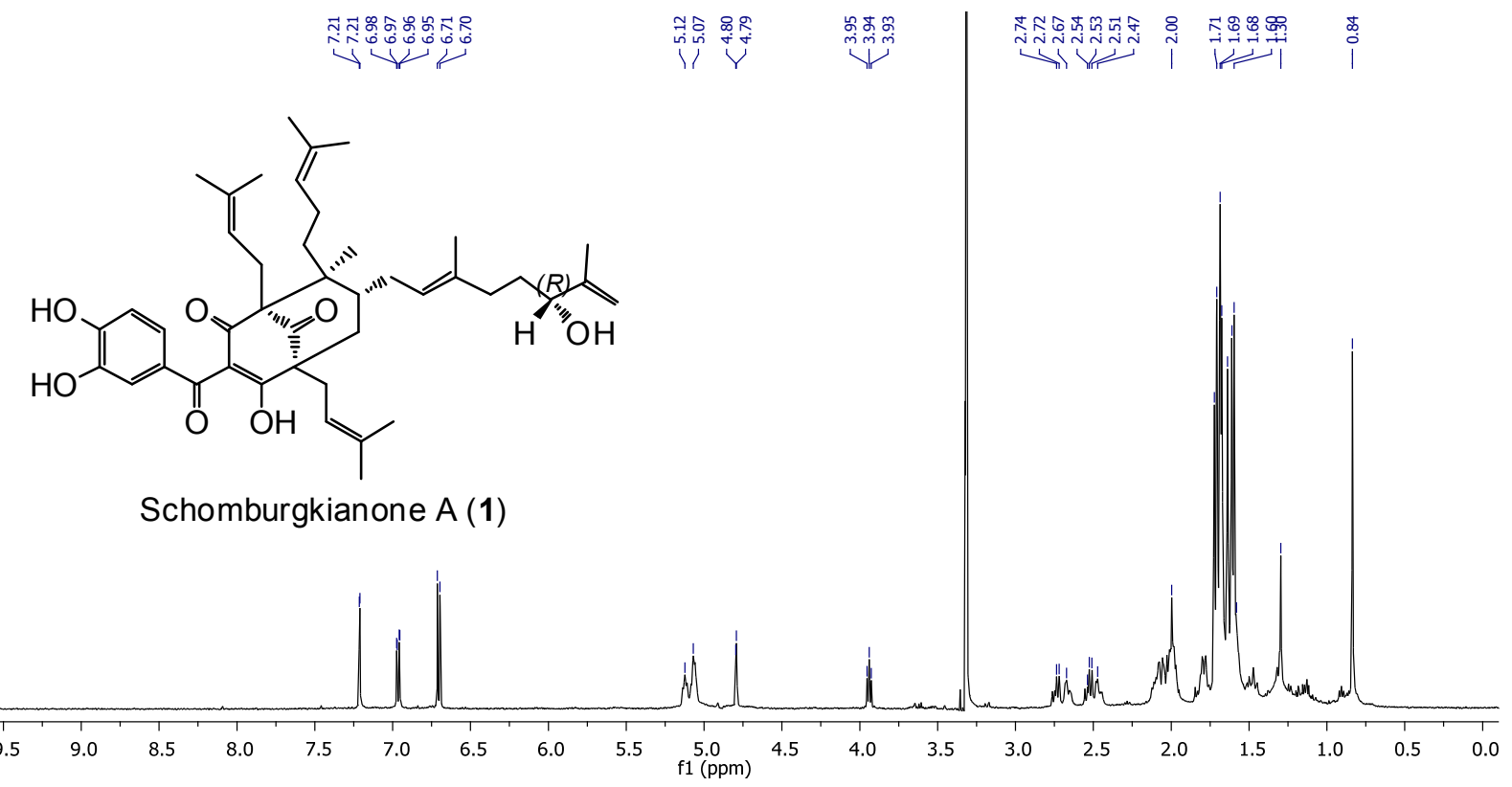

Figure S5. ${ }^{1} \mathrm{H}$ NMR spectrum $\left(500 \mathrm{MHz}\right.$, methanol- $d_{4}+0.1 \%$ TFA- $d$ ) of 1
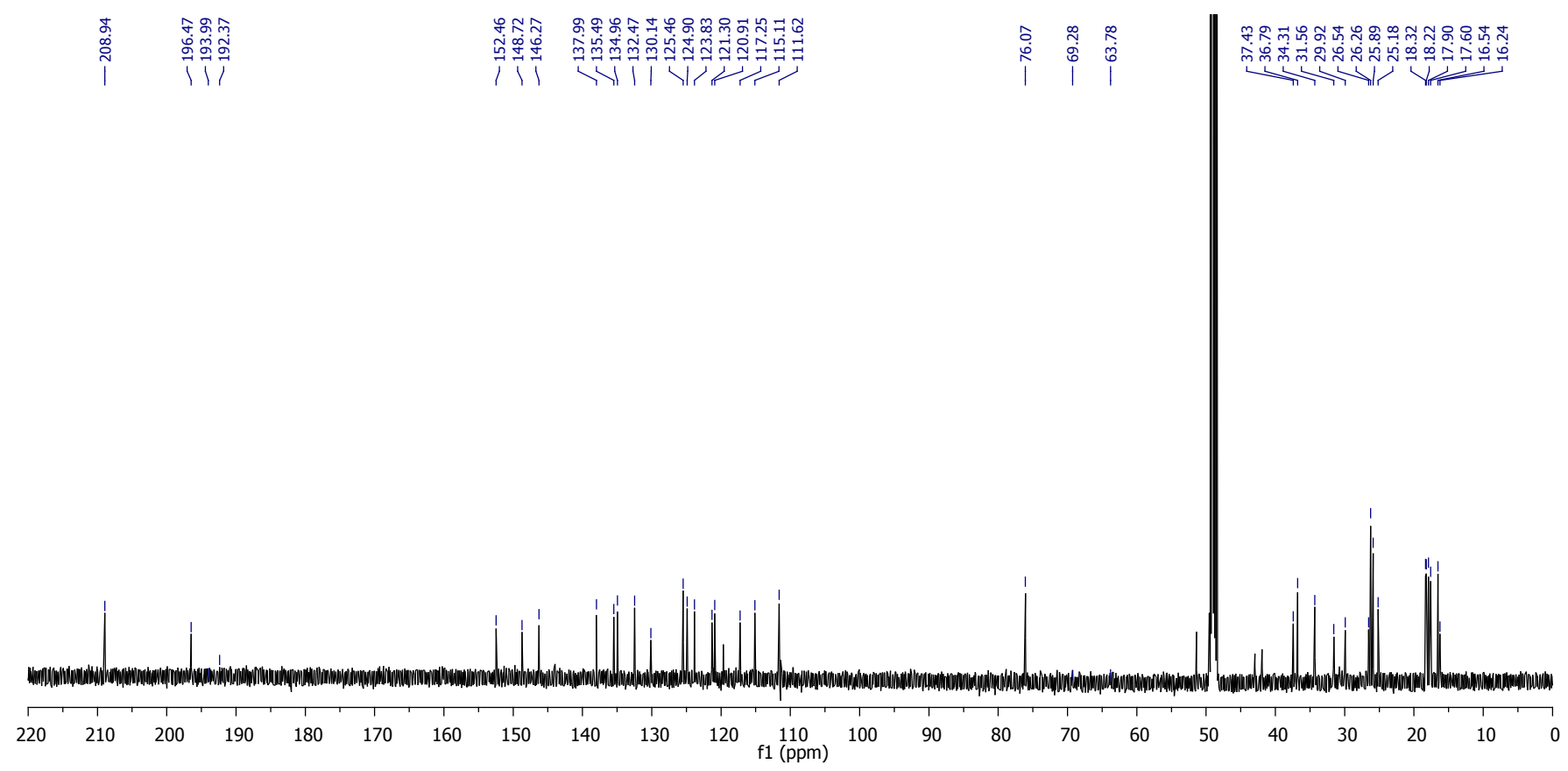

Figure S6. ${ }^{13} \mathrm{C}$ NMR spectrum $\left(125 \mathrm{MHz}\right.$, methanol- $d_{4}+0.1 \%$ TFA- $d$ ) of $\mathbf{1}$ 

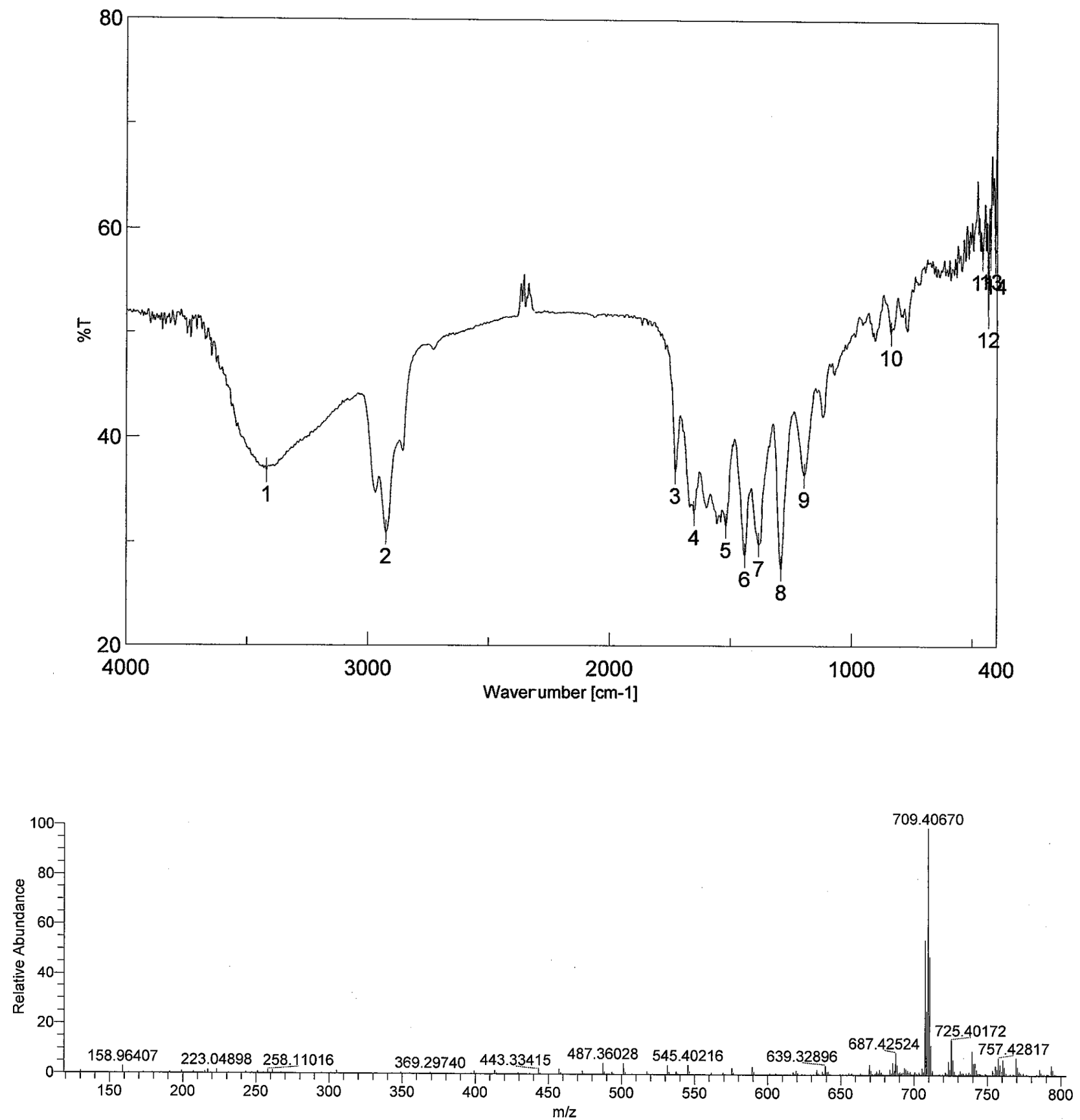

GS11 \#17-20 RT: 0.37-0.41 AV: 2 SB: 8 0.68-1.04 NL: 8.10E4 T: FTMS $\{1,1\}+p$ ESI Full ms $[120.00-1000.00]$

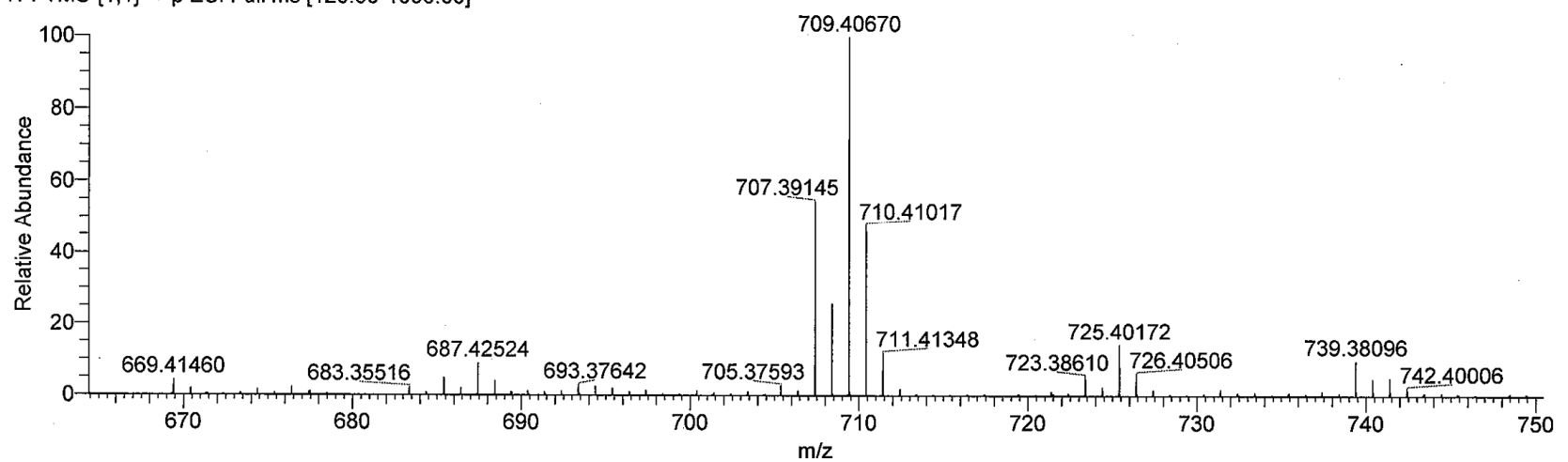

Figure S7. IR and MS spectra of 2 


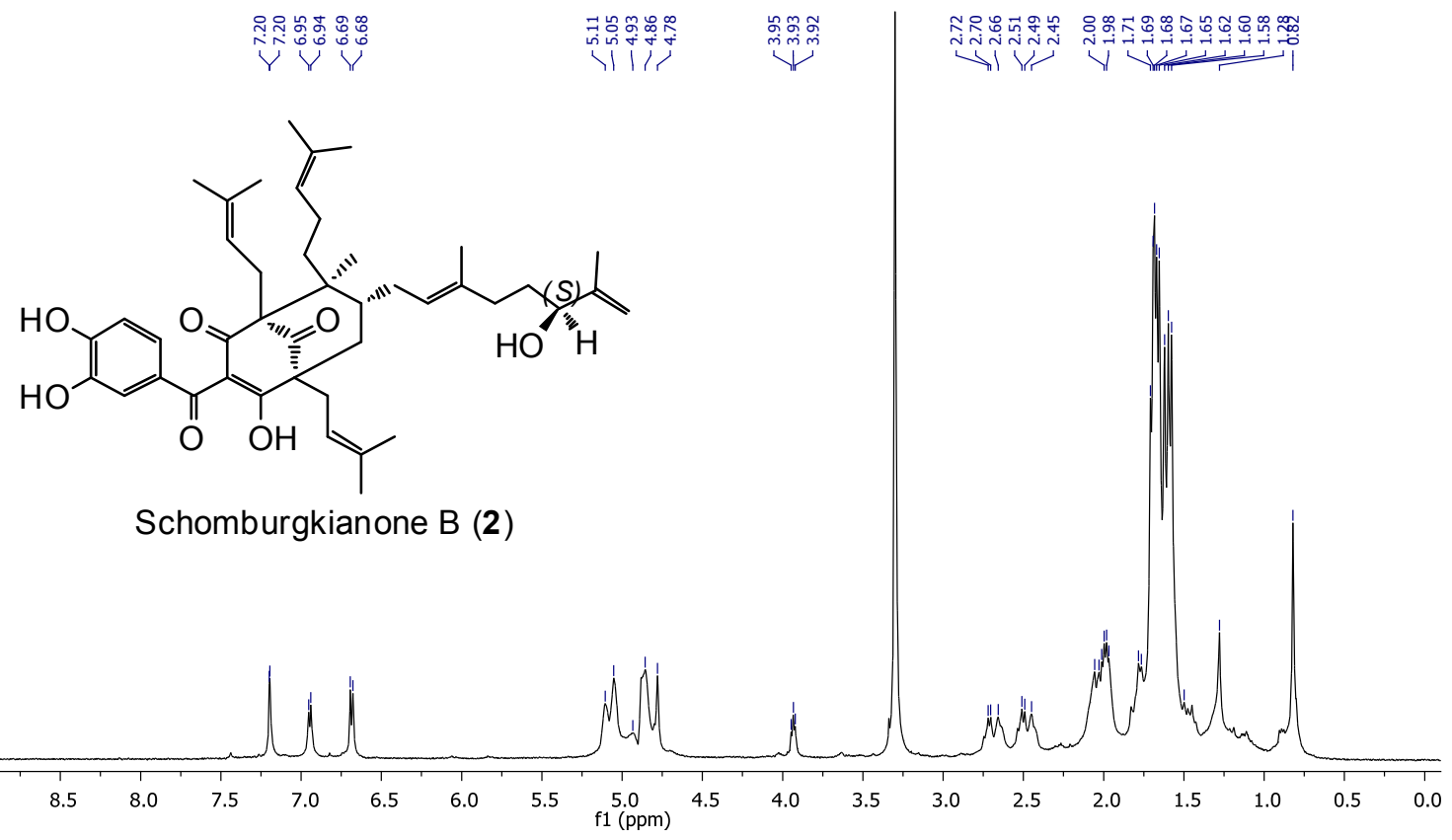

Figure S8. ${ }^{1} \mathrm{H}$ NMR spectrum $\left(500 \mathrm{MHz}\right.$, methanol- $d_{4}+0.1 \%$ TFA- $d$ ) of 2

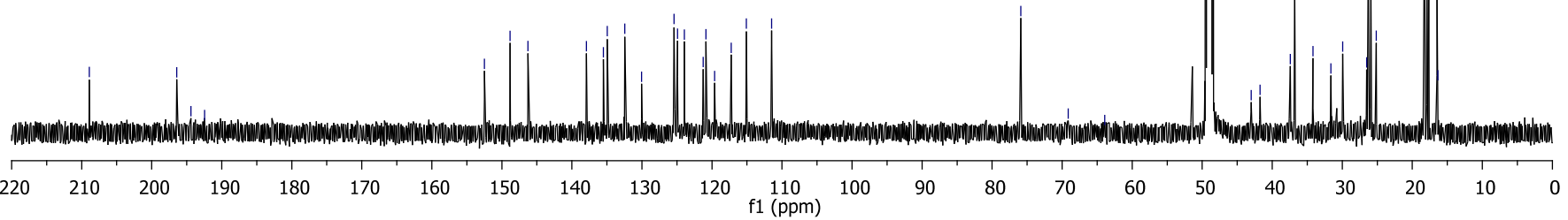

Figure S9. ${ }^{13} \mathrm{C}$ NMR spectrum $\left(125 \mathrm{MHz}\right.$, methanol- $d_{4}+0.1 \%$ TFA- $d$ ) of 2 

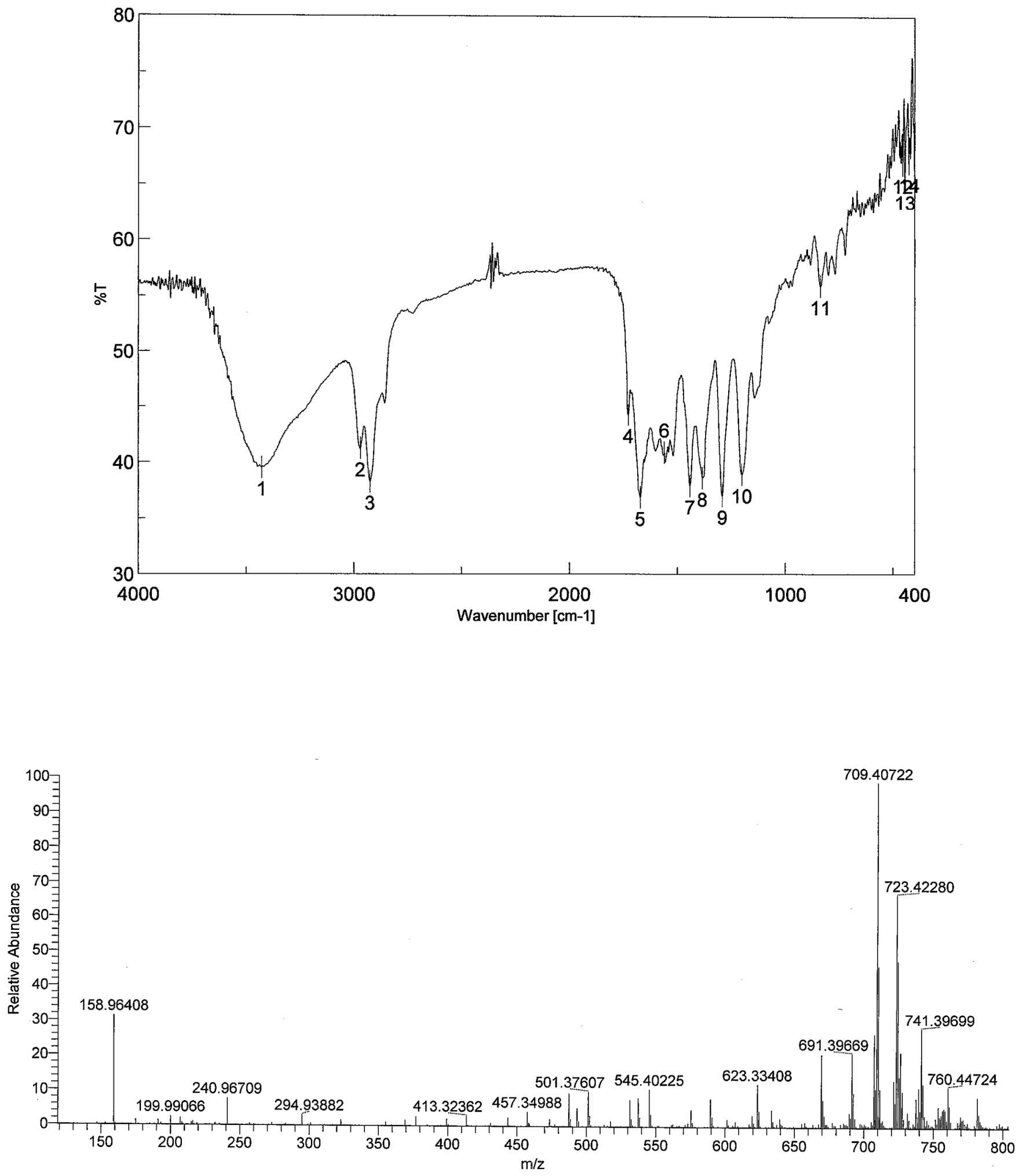

Figure S10. IR and MS spectra of 3 


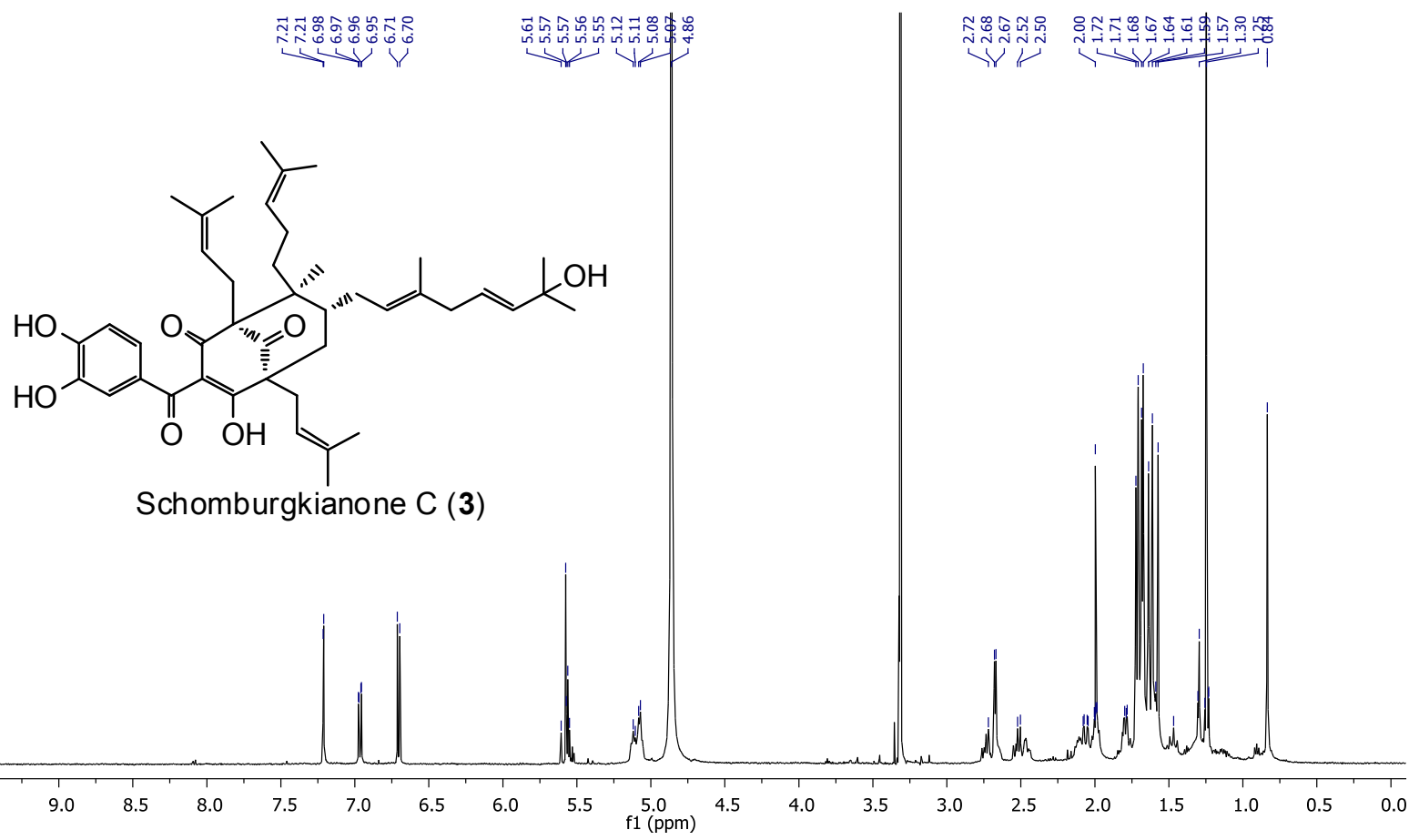

Figure S11. ${ }^{1} \mathrm{H}$ NMR spectrum $\left(500 \mathrm{MHz}\right.$, methanol- $d_{4}+0.1 \%$ TFA- $d$ ) of 3

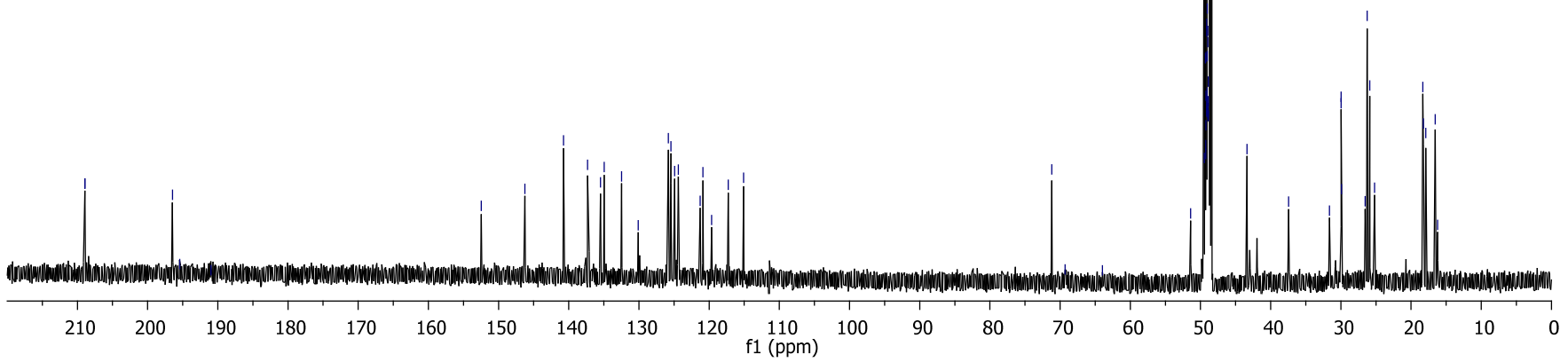

Figure S12. ${ }^{13} \mathrm{C}$ NMR spectrum (125 MHz, methanol- $d_{4}+0.1 \%$ TFA- $d$ ) of 3 

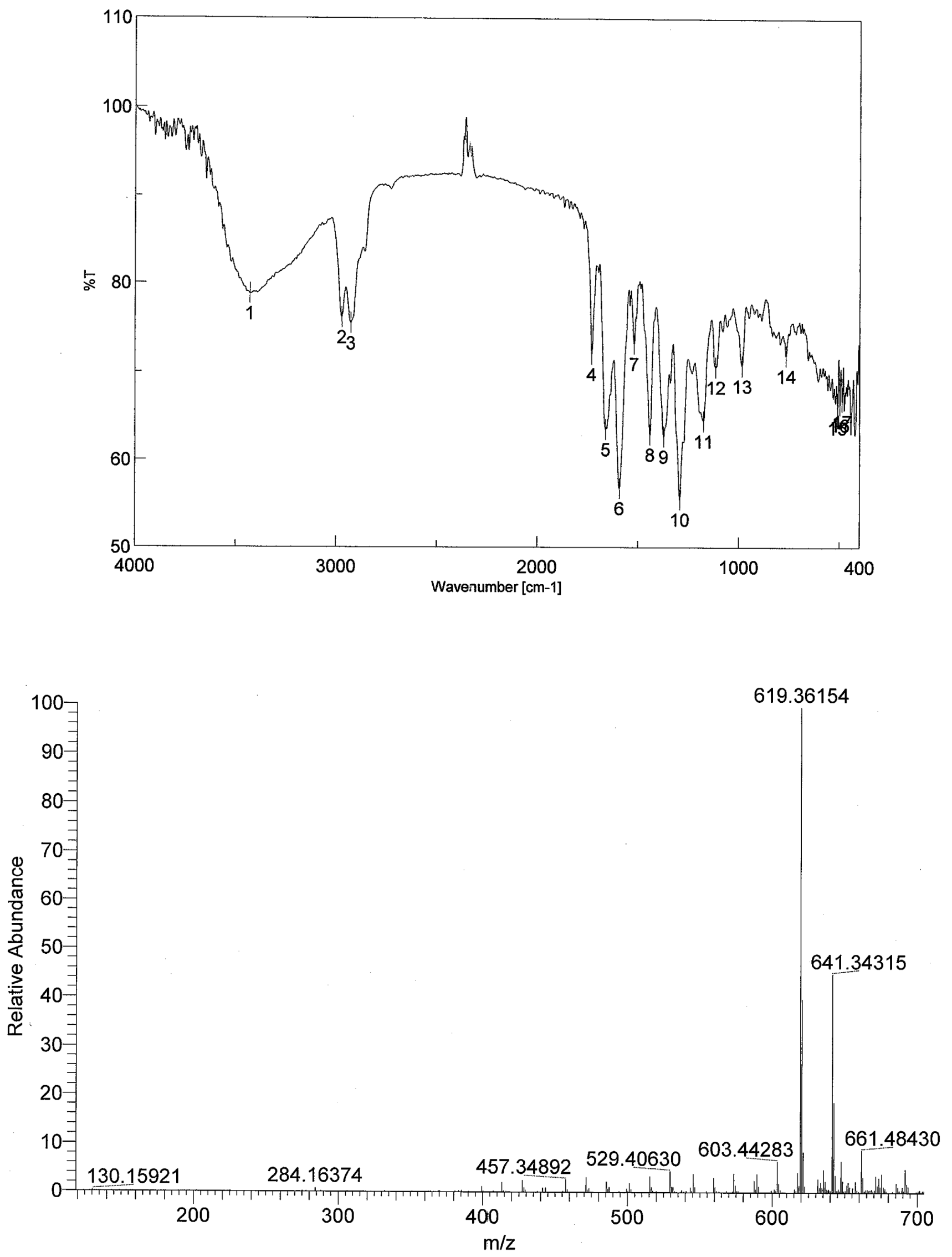

Figure S13. IR and MS spectra of 4 


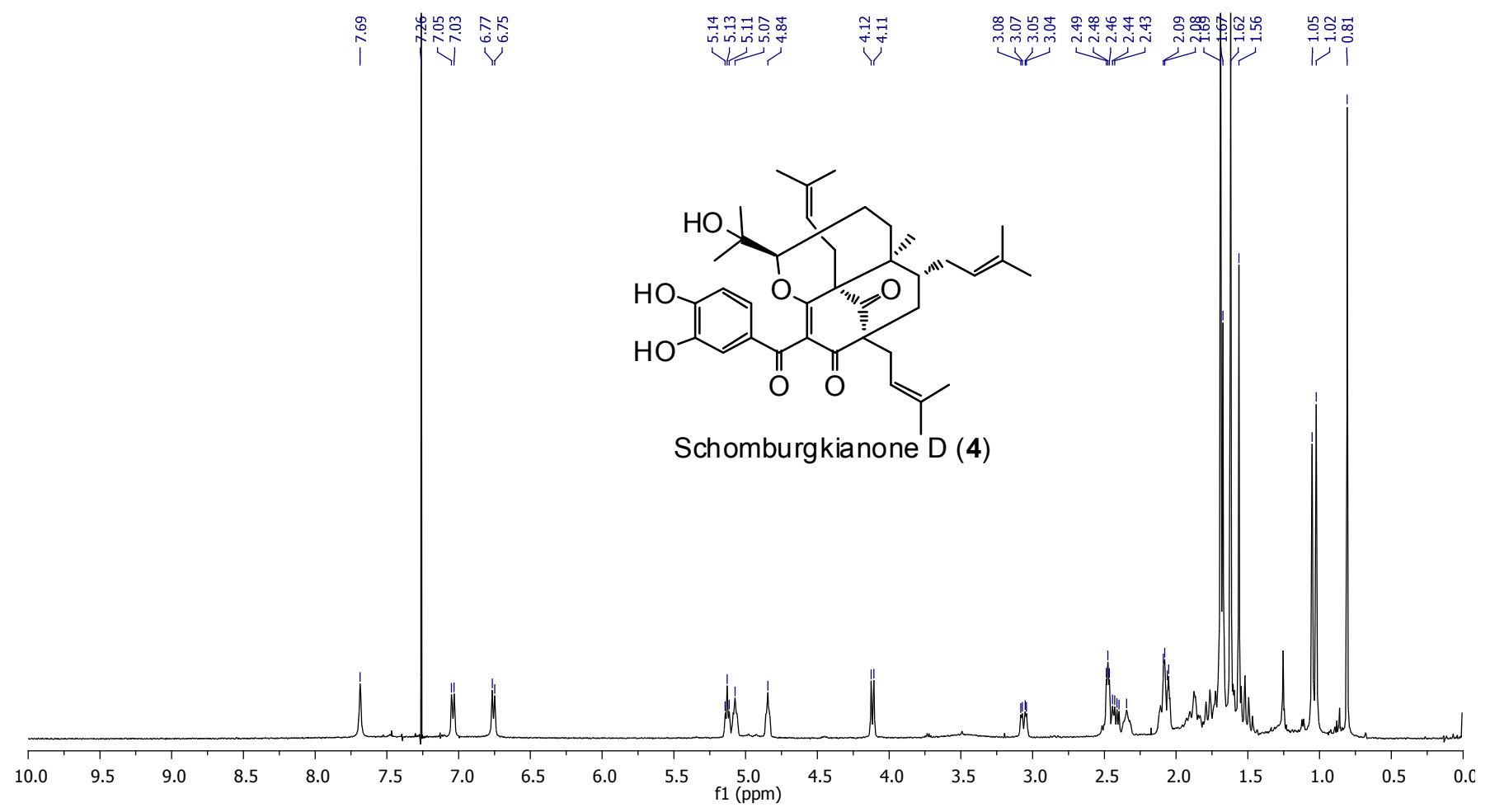

Figure S14. ${ }^{1} \mathrm{H}$ NMR spectrum $\left(500 \mathrm{MHz}, \mathrm{CDCl}_{3}\right)$ of 4

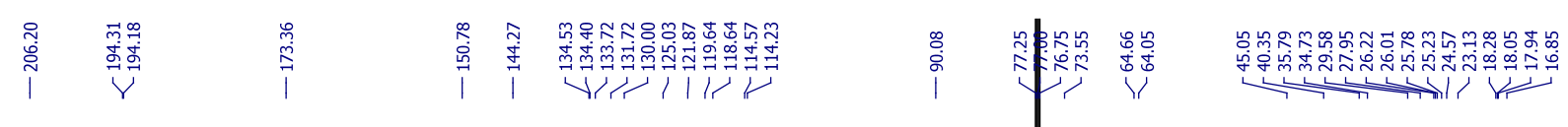

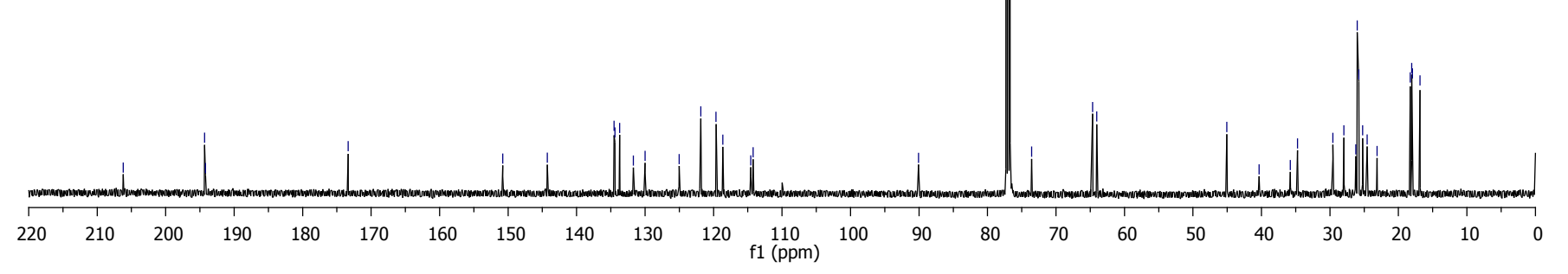

Figure S15. ${ }^{13} \mathrm{C}$ NMR spectrum $\left(125 \mathrm{MHz}, \mathrm{CDCl}_{3}\right)$ of 4 

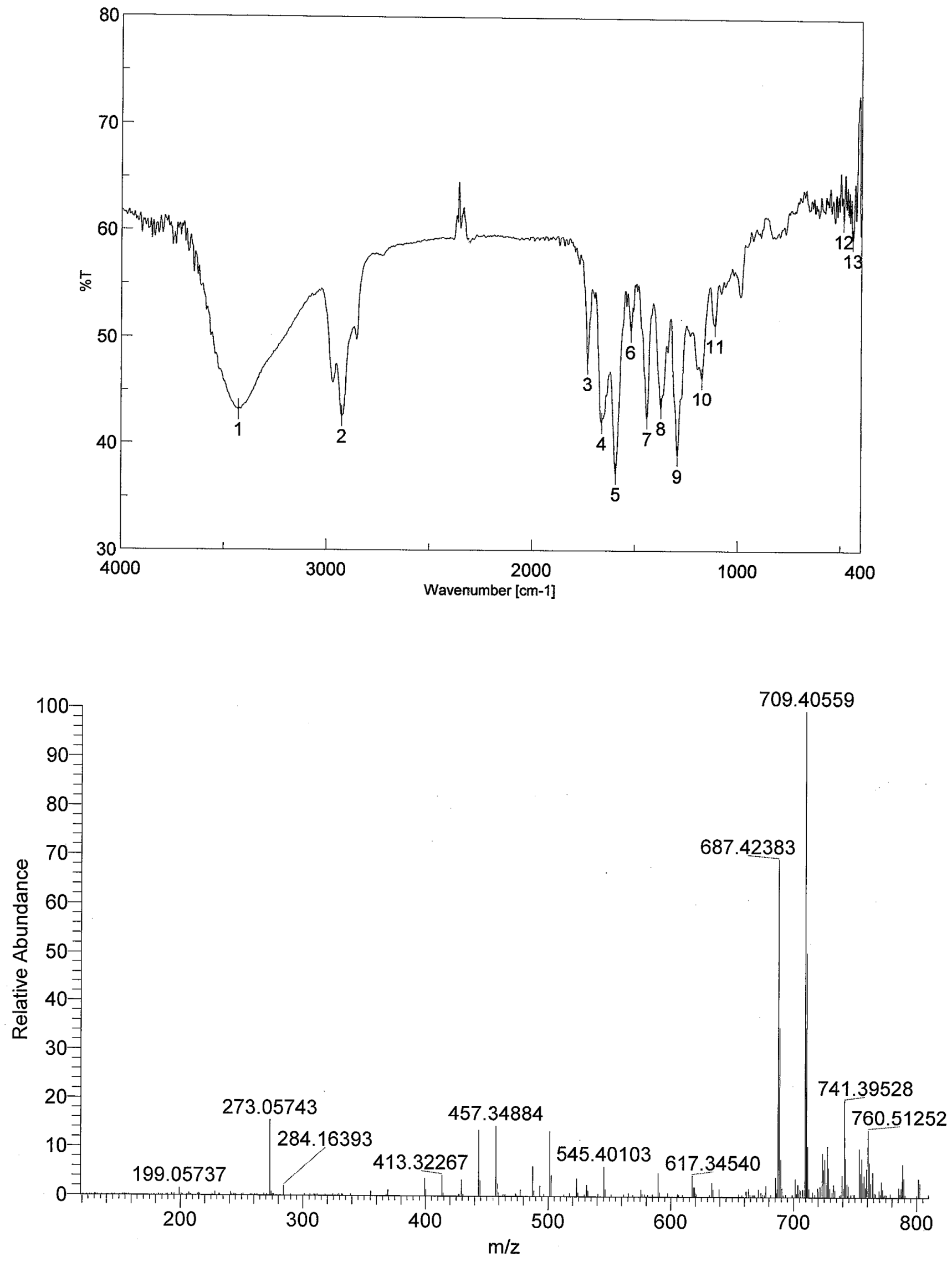

Figure S16. IR and MS spectra of 5 


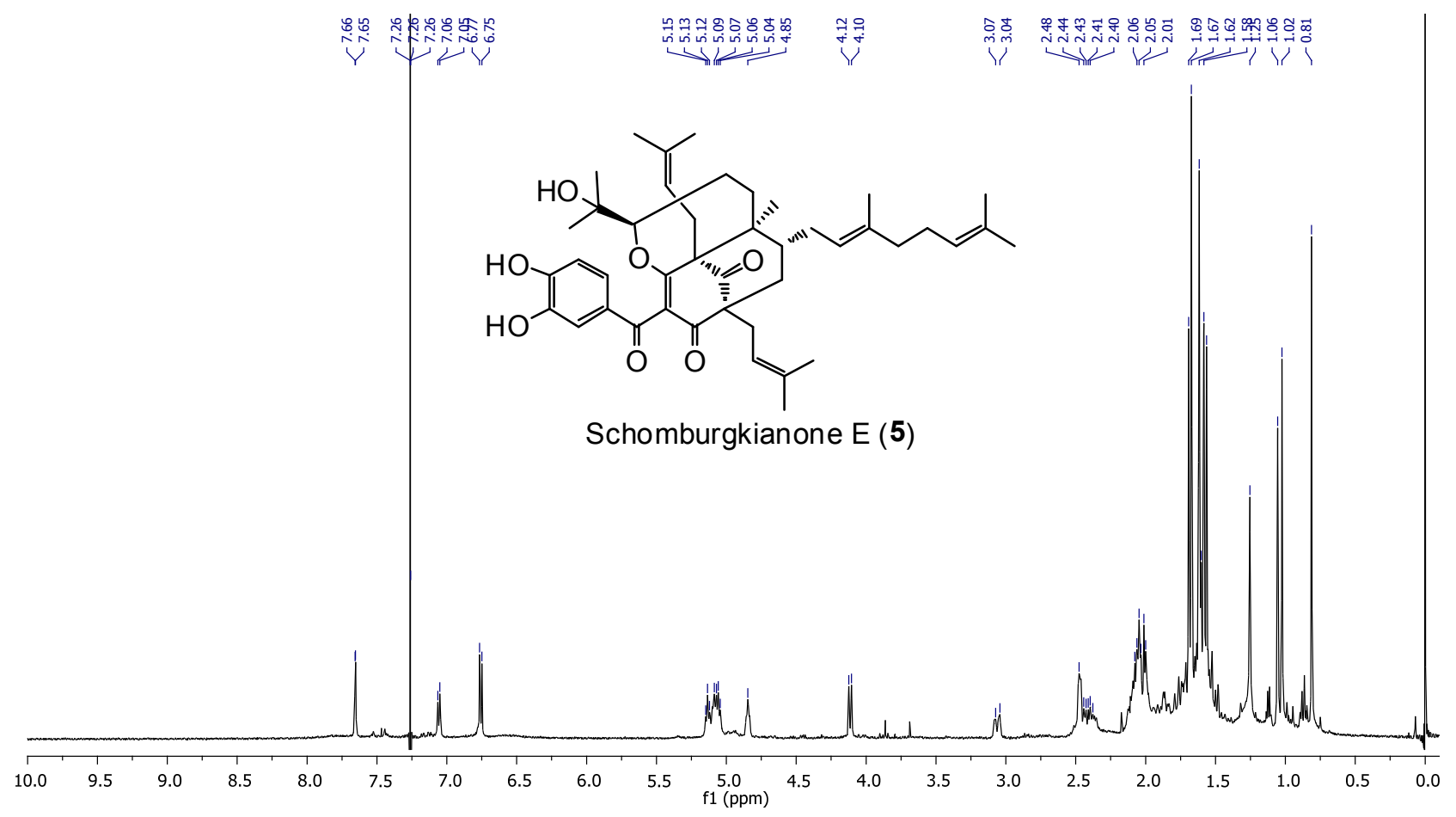

Figure S17. ${ }^{1} \mathrm{H}$ NMR spectrum $\left(500 \mathrm{MHz}, \mathrm{CDCl}_{3}\right)$ of 5

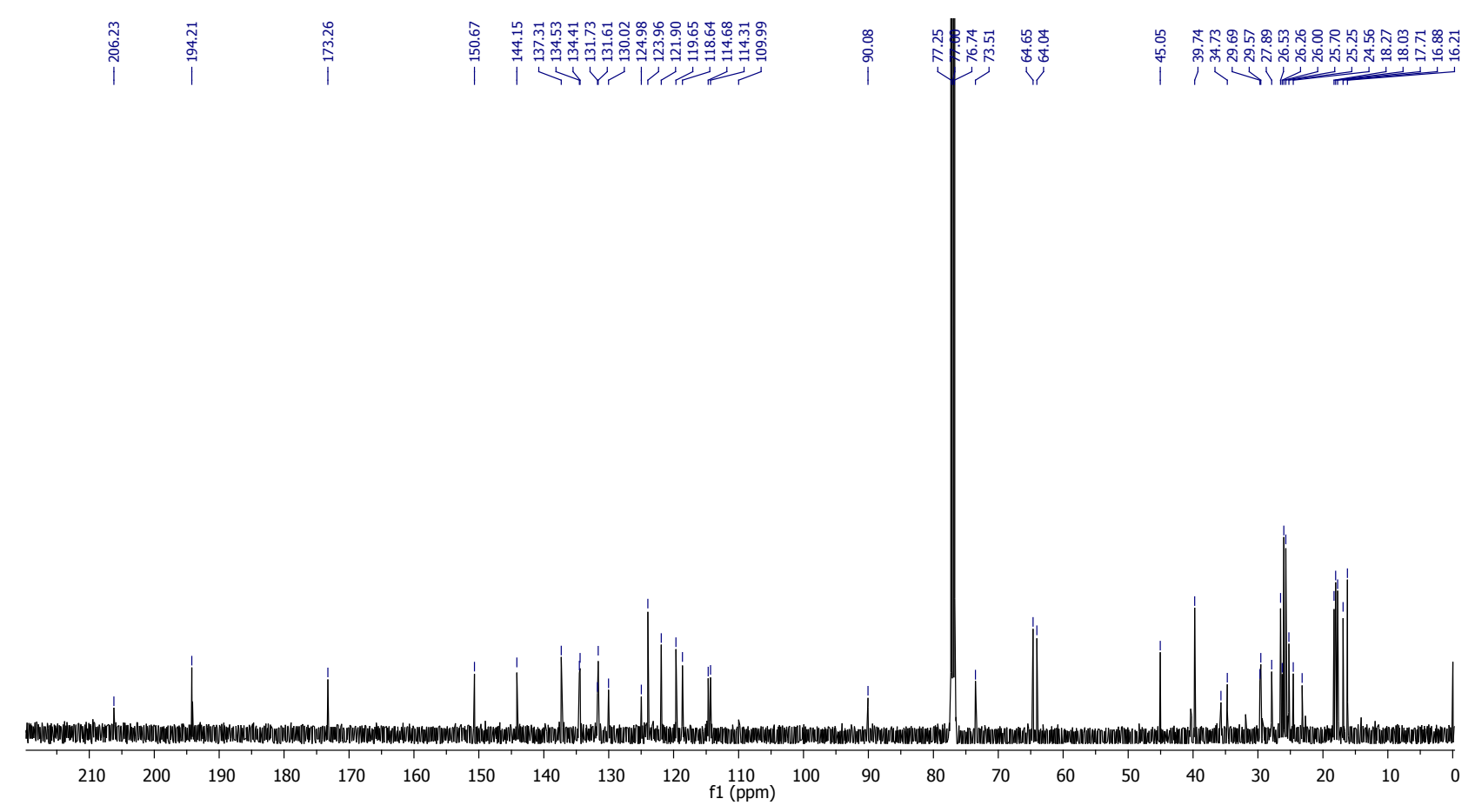

Figure S18. ${ }^{13} \mathrm{C}$ NMR spectrum $\left(125 \mathrm{MHz}, \mathrm{CDCl}_{3}\right)$ of $\mathbf{5}$ 

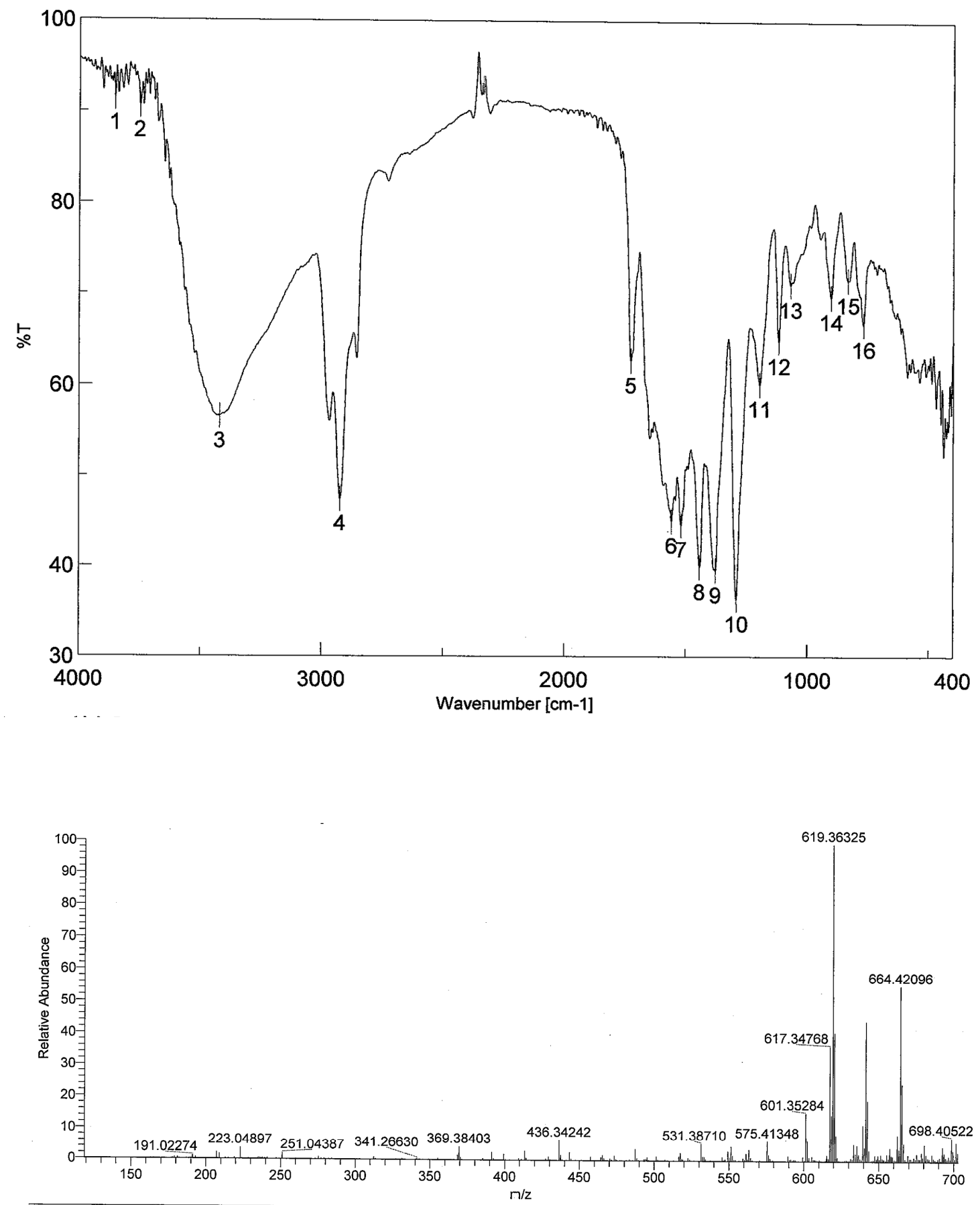

YY_GS14\#15-17 RT: 0.31-0.36 AV: 2 SB: 5 0.05-0.12, 0.44-0.54 NL: 6.32E4

T: FTMS $\{1,1\}+p$ ESI Full ms [120.00-1000.00]

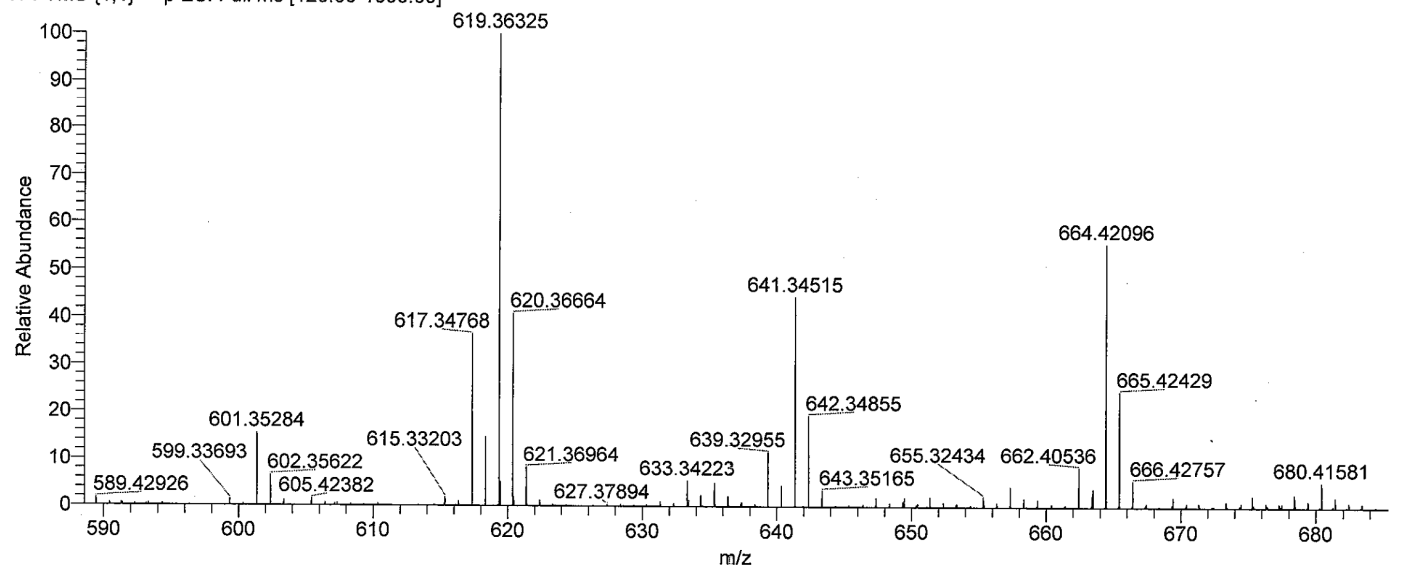

Figure S19. IR and MS spectra of 6 


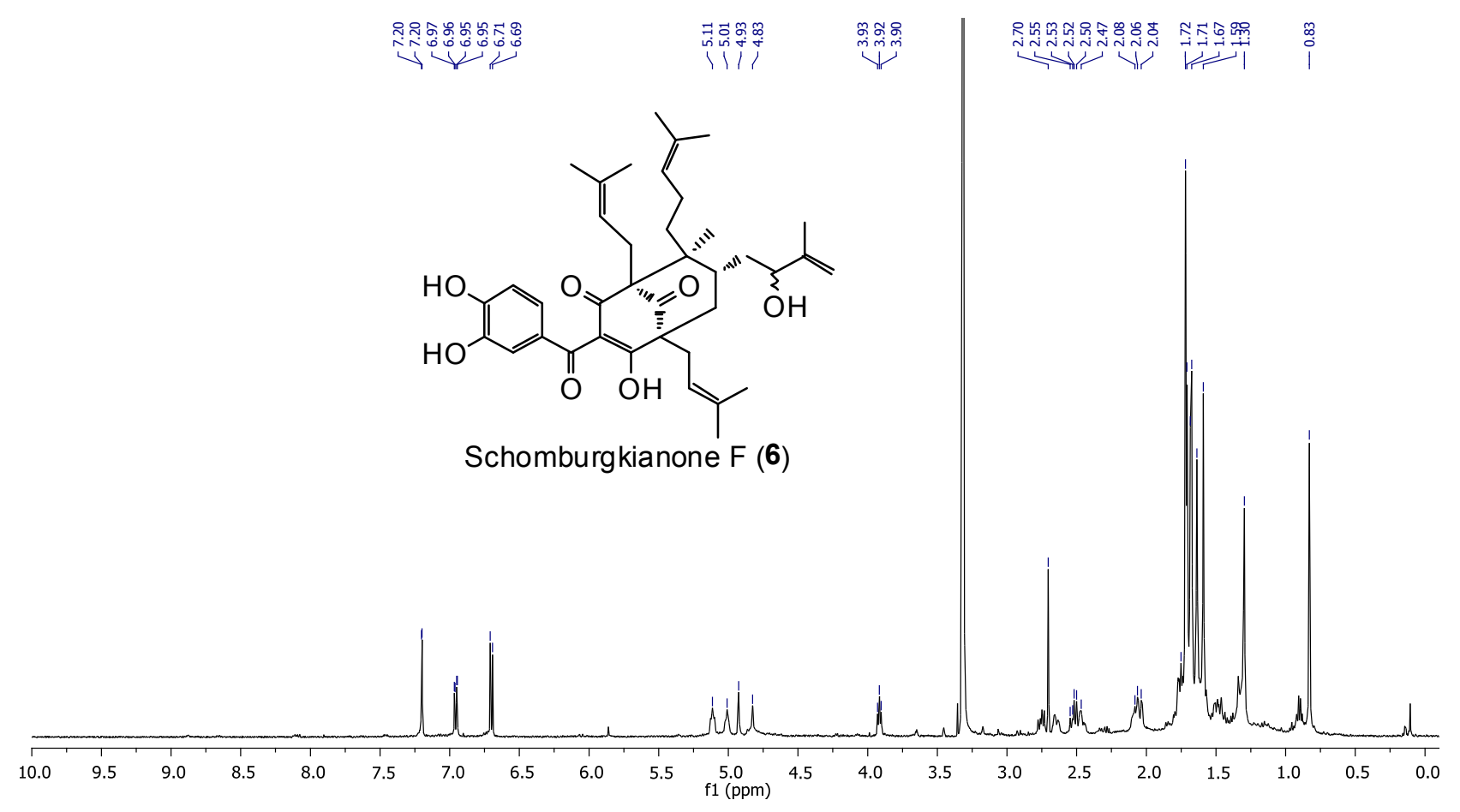

Figure S20. ${ }^{1} \mathrm{H}$ NMR spectrum $\left(500 \mathrm{MHz}\right.$, methanol- $d_{4}+0.1 \%$ TFA- $d$ ) of 6

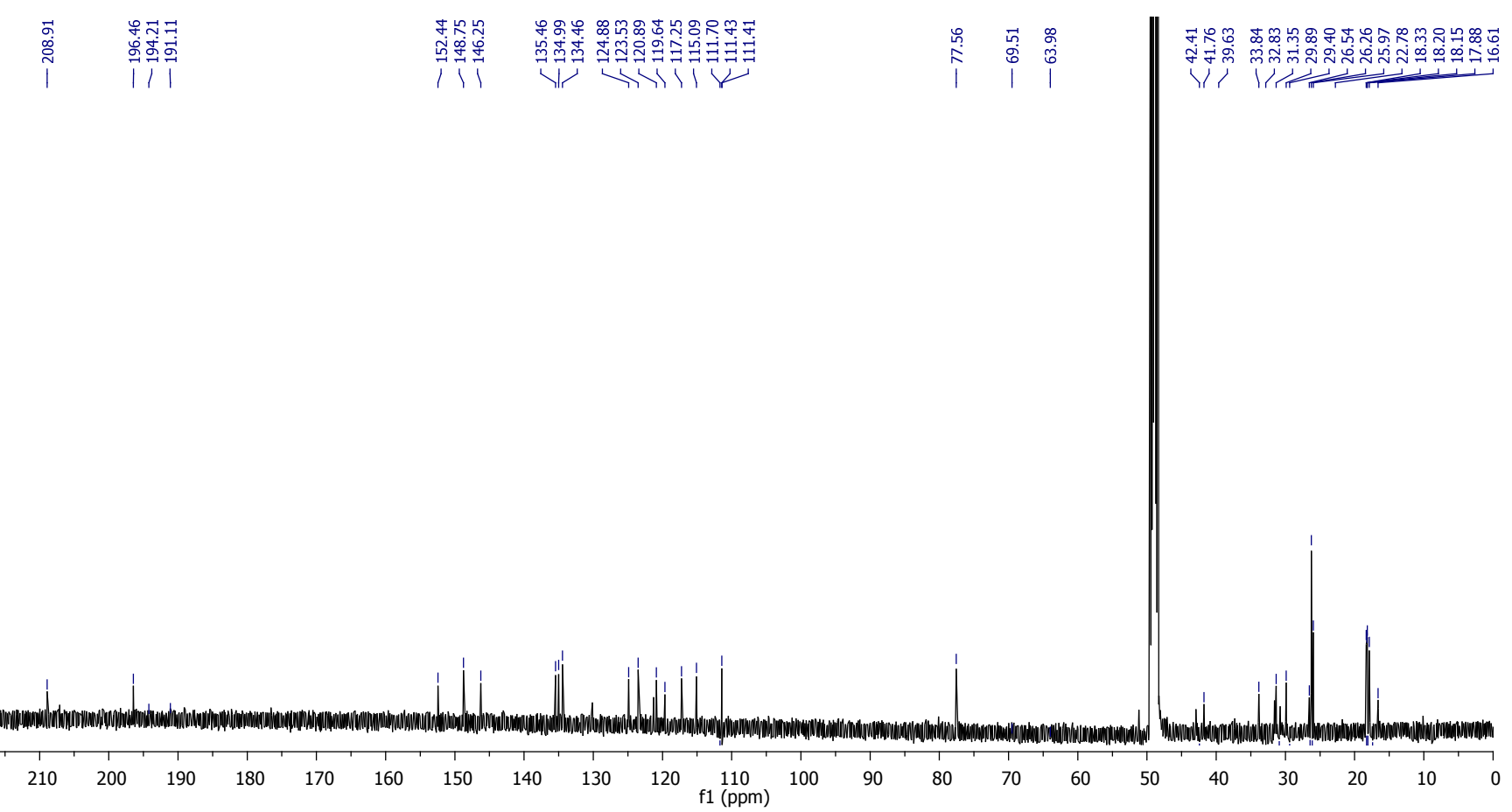

Figure S21. ${ }^{13} \mathrm{C}$ NMR spectrum $\left(125 \mathrm{MHz}\right.$, methanol- $d_{4}+0.1 \%$ TFA- $d$ ) of 6 

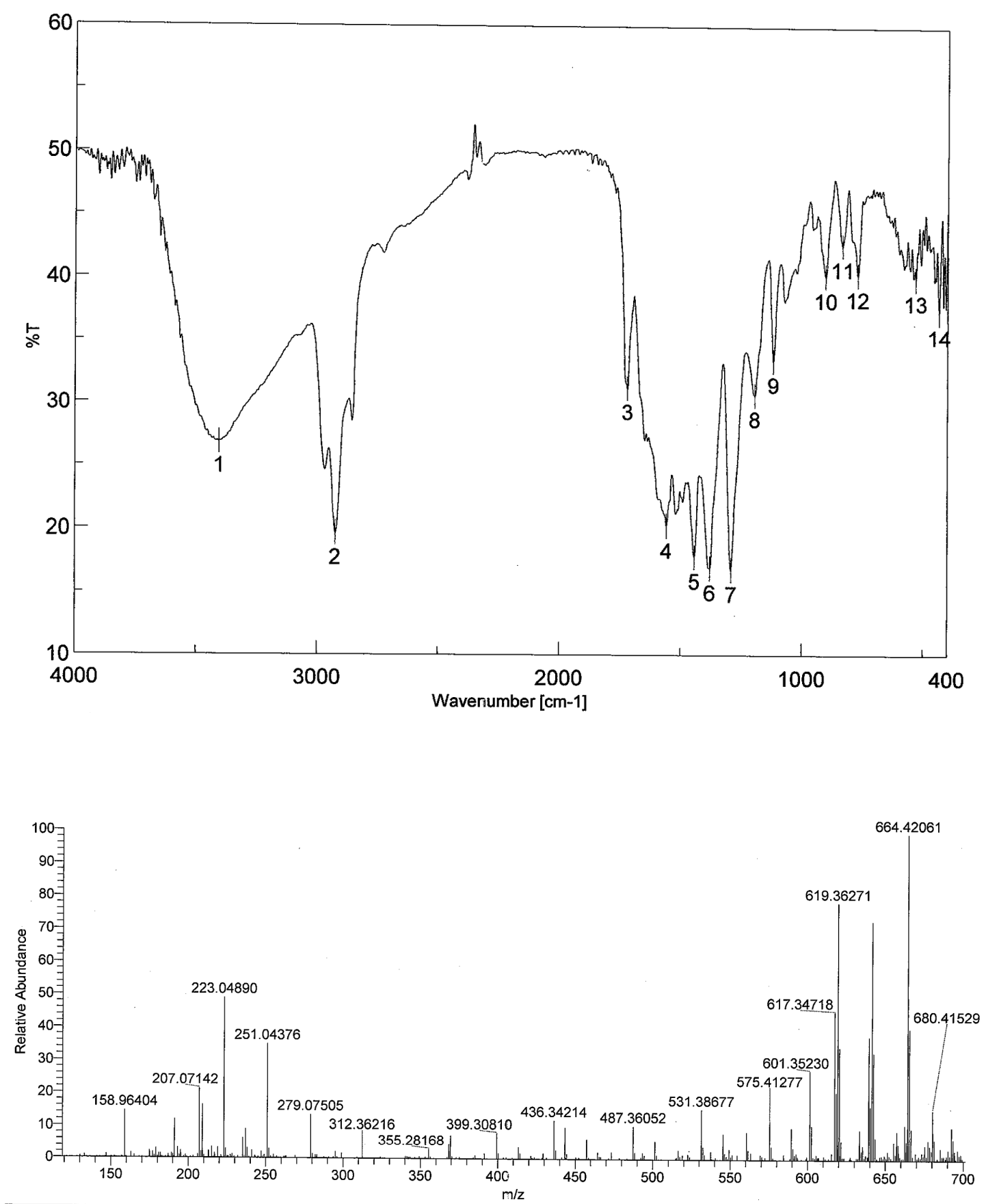

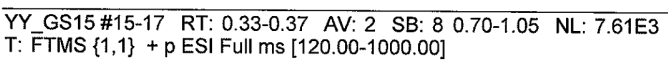

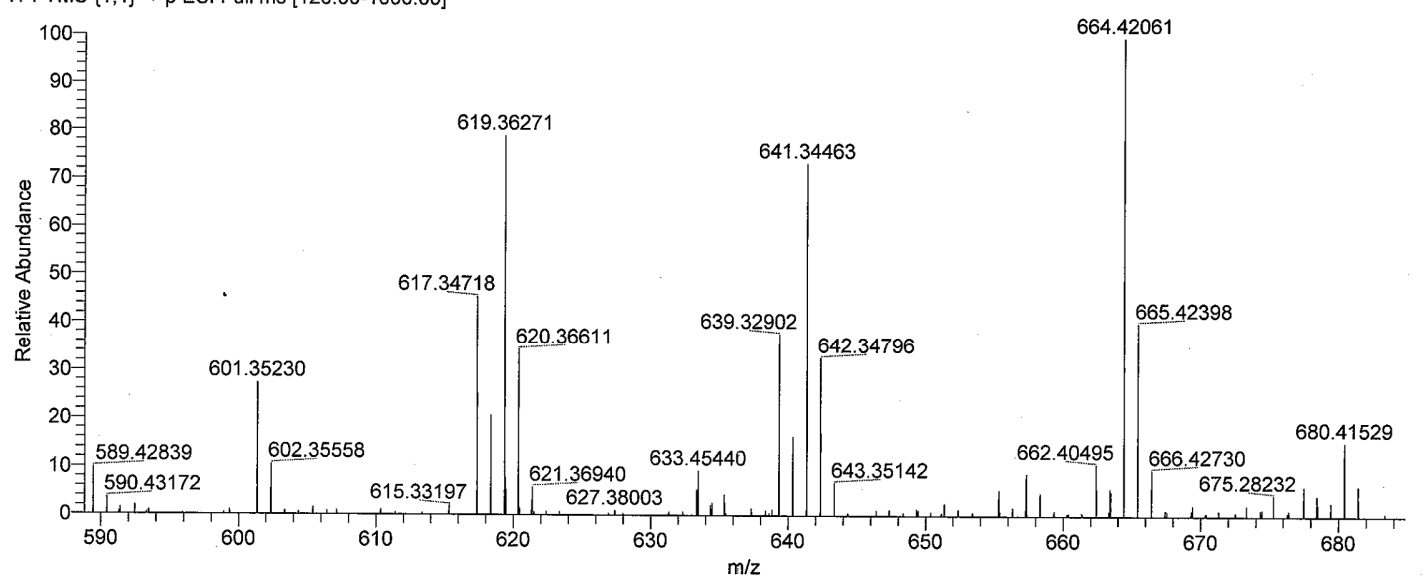

Figure S22. IR and MS spectra of 7 


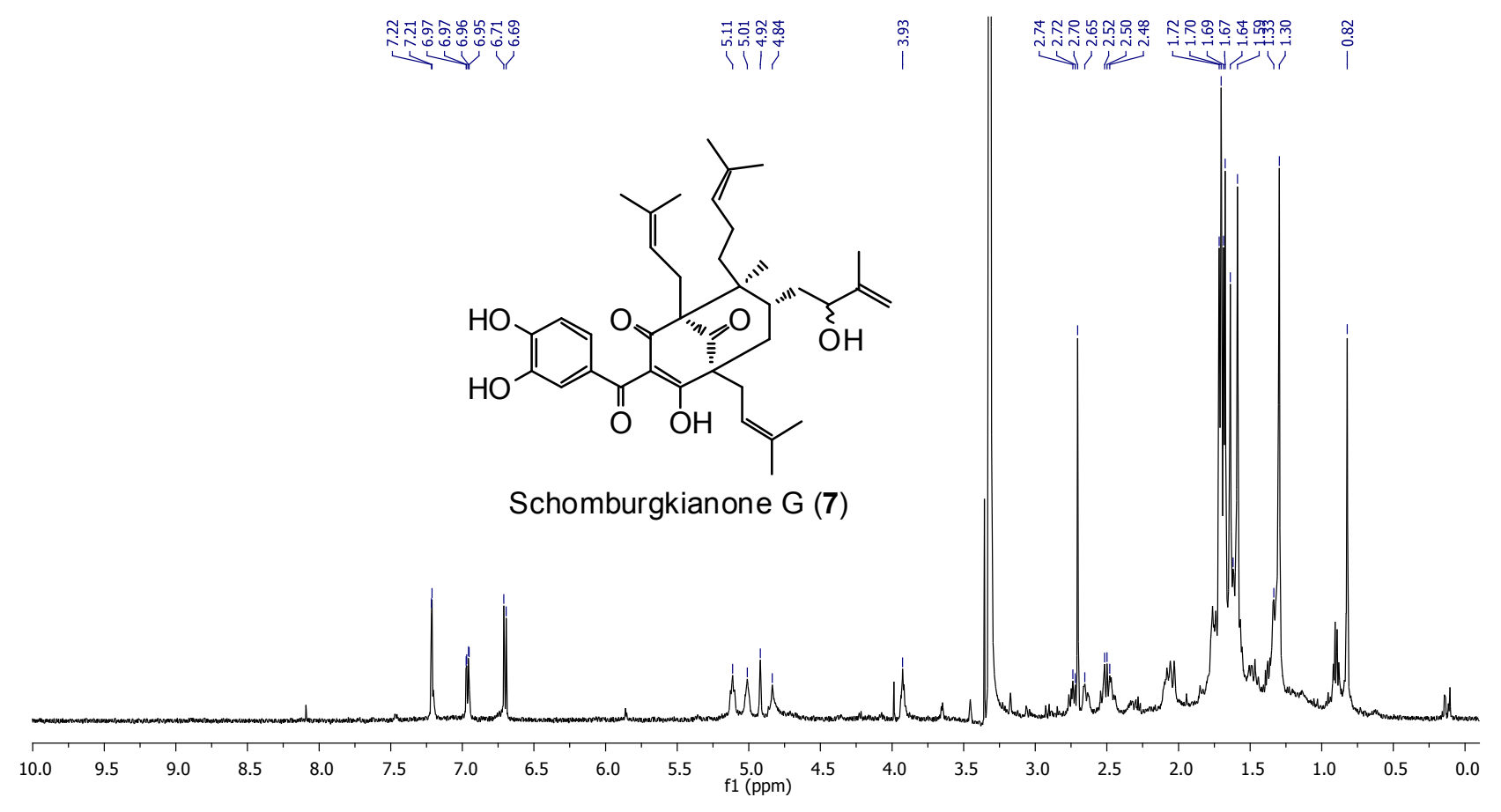

Figure S23. ${ }^{1} \mathrm{H}$ NMR spectrum $\left(500 \mathrm{MHz}\right.$, methanol- $d_{4}+0.1 \%$ TFA- $d$ ) of 7
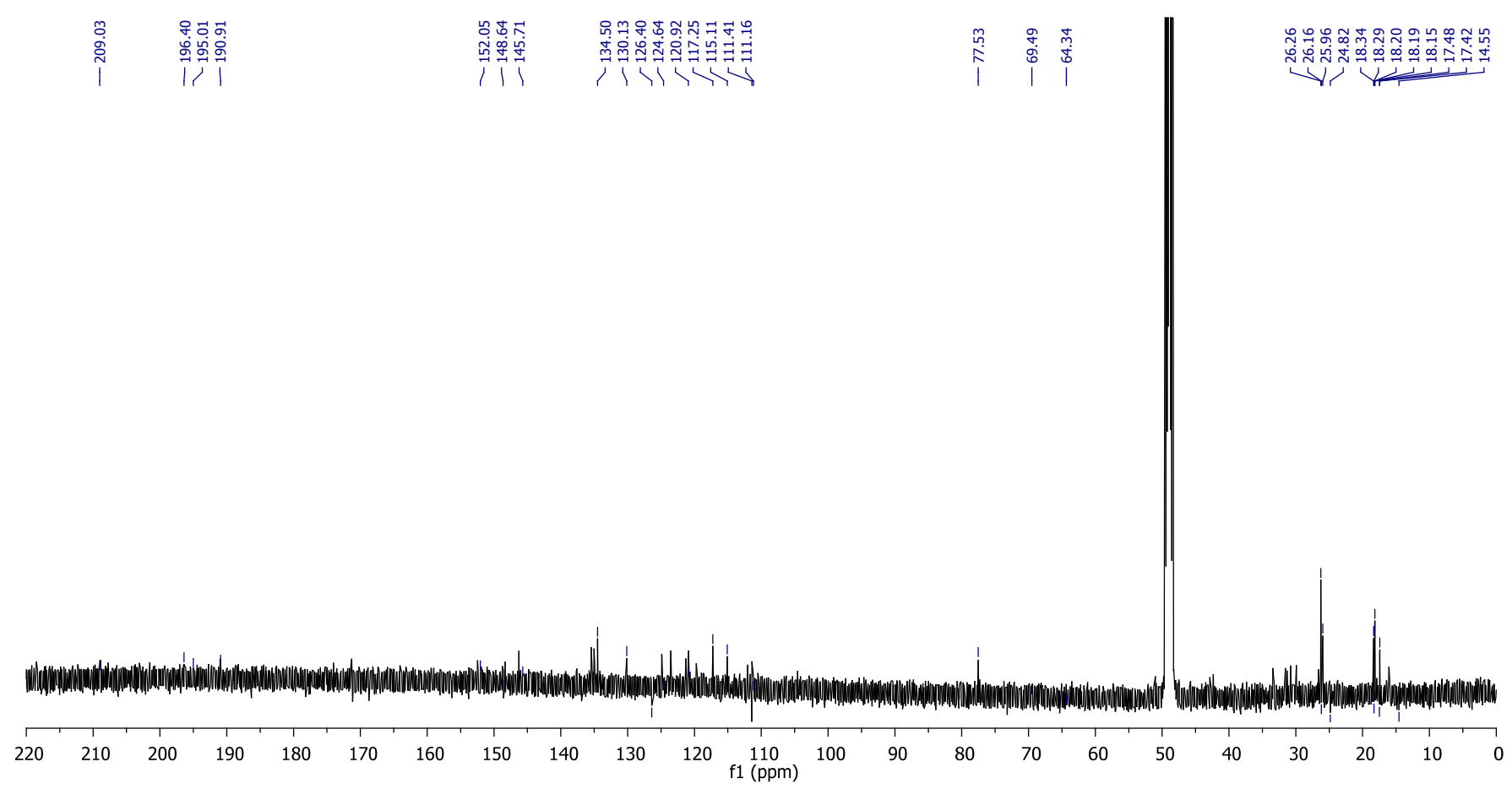

Figure S24. ${ }^{13} \mathrm{C}$ NMR spectrum $\left(125 \mathrm{MHz}\right.$, methanol- $d_{4}+0.1 \%$ TFA- $d$ ) of 7 

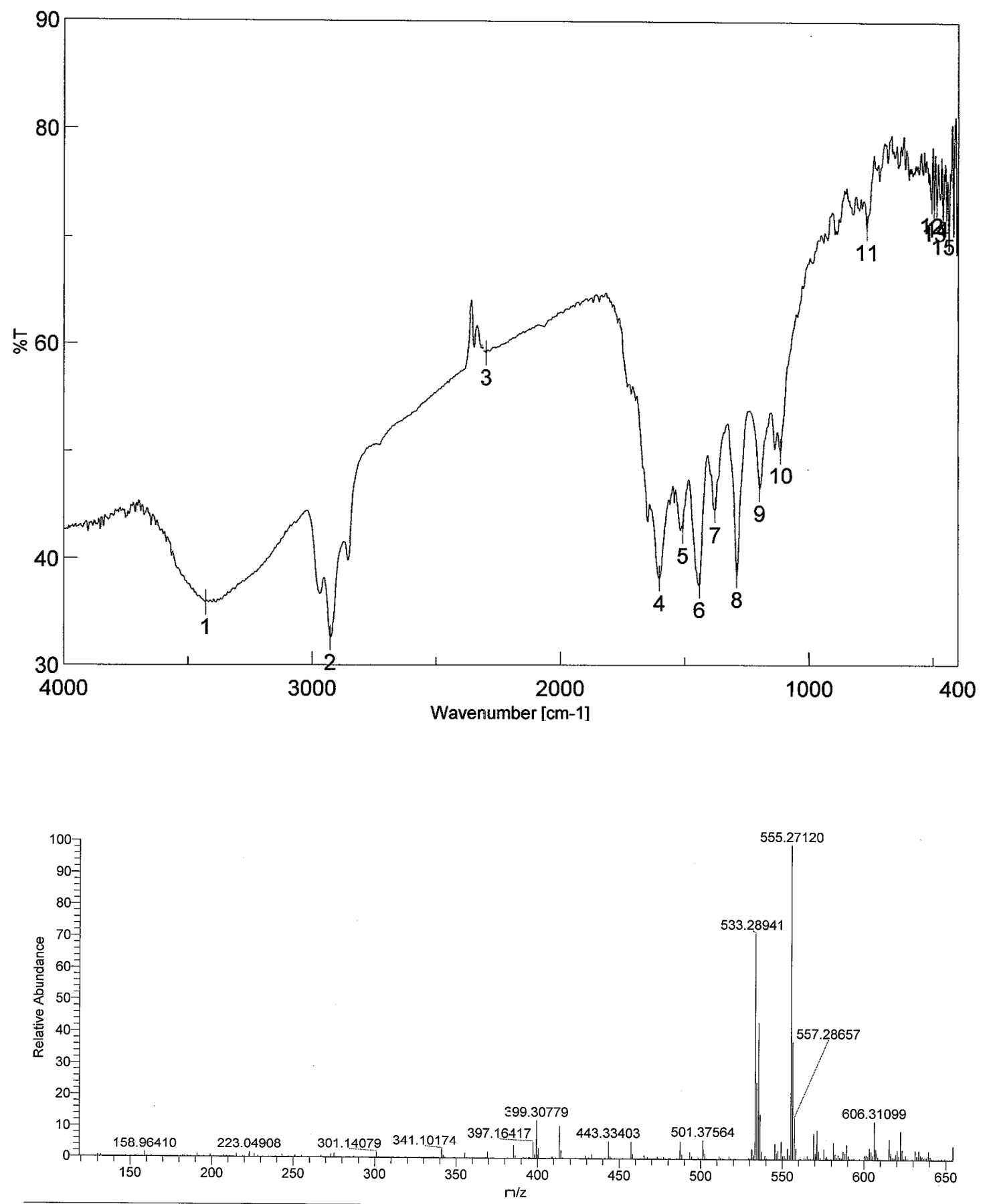

GS10\#17-19 RT: 0.38-0.42 AV: 2 SB: 8 0.69-1.05 NL: 1.83E4

T: FTMS $\{1,1\}+p$ ESI Full ms [120.00-1000.00]

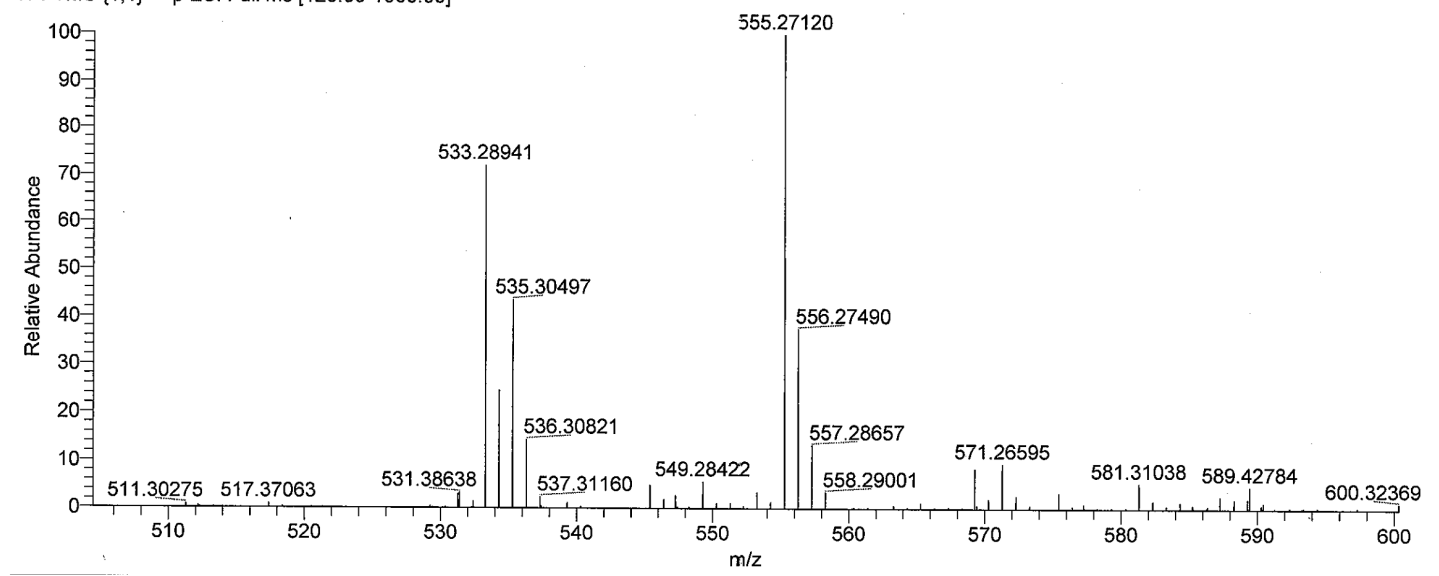

Figure S25. IR and MS spectra of 8 


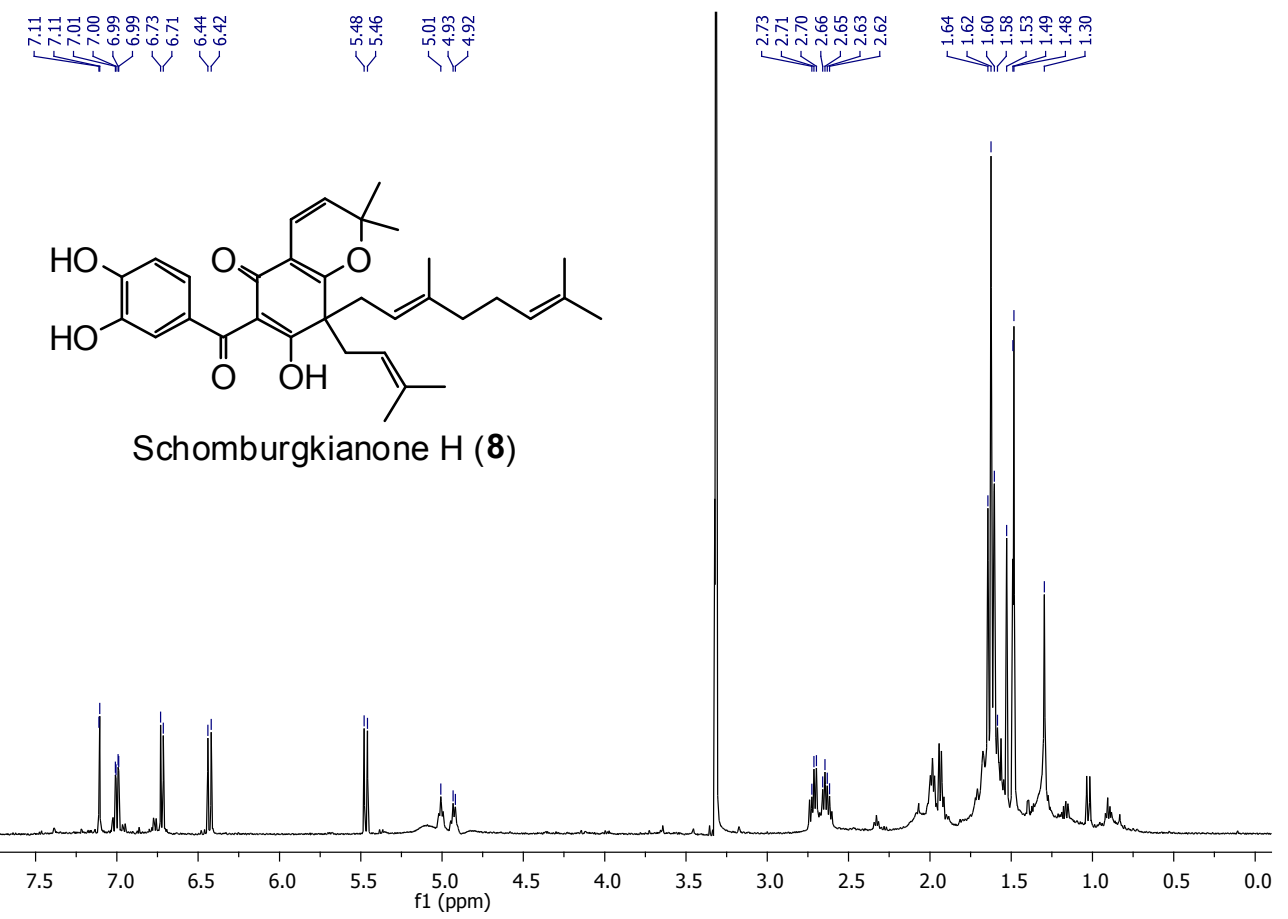

Figure S26. ${ }^{1} \mathrm{H}$ NMR spectrum (500 MHz, methanol- $d_{4}+0.1 \%$ TFA- $d$ ) of 8

$$
1
$$

Figure S27. ${ }^{13} \mathrm{C}$ NMR spectrum $\left(125 \mathrm{MHz}\right.$, methanol- $d_{4}+0.1 \%$ TFA- $d$ ) of 8 